\title{
VERTICES OF GIVEN DEGREE IN SERIES-PARALLEL GRAPHS
}

\author{
MICHAEL DRMOTA, OMER GIMENEZ, AND MARC NOY
}

\begin{abstract}
We show that the number of vertices of a given degree $k$ in several kinds of series-parallel labelled graphs of size $n$ satisfy a central limit theorem with mean and variance proportional to $n$, and quadratic exponential tail estimates. We further prove a corresponding theorem for the number of nodes of degree two in labelled planar graphs. The proof method is based on generating functions and singularity analysis. In particular we need systems of equations for multivariate generating functions and transfer results for singular representations of analytic functions.
\end{abstract}

\section{Statement of MAin RESUlts}

A graph is series-parallel if it does not contain the complete graph $K_{4}$ as a minor; equivalently, if it does not contain a subdivision of $K_{4}$. Since both $K_{5}$ and $K_{3,3}$ contain a subdivision of $K_{4}$, by Kuratowski's theorem a series-parallel graph is planar. An outerplanar graph is a planar graph that can be embedded in the plane so that all vertices are incident to the outer face. They are characterized as those graphs not containing a minor isomorphic to (or a subdivision of) either $K_{4}$ or $K_{2,3}$. These are important subfamilies of planar graphs, as they are much simpler but often they already capture the essential structural properties of planar graphs. In particular, they are used as a natural first benchmark for many algorithmic problems and conjectures related to planar graphs

The purpose of this paper is to study the number of vertices of given degree in certain classes of labelled planar graphs. In particular, we study labelled outerplanar graphs and series-parallel graphs; in what follows, all graphs are labelled.

In order to state our results we introduce the notion of the degree distribution of a random outerplanar graph (the definition for series-parallel graphs is exactly the same). For every $n$ we consider the class of all vertex labelled outerplanar graphs with $n$ vertices. Let $D_{n}$ denote the degree of a randomly chosen vertex in this class of graphs ${ }^{1}$. Then we say that this class of graphs has a degree distribution if there exist non-negative numbers $d_{k}$ with $\sum_{k \geq 0} d_{k}=1$ such that for all $k$

$$
\lim _{n \rightarrow \infty} \operatorname{Pr}\left\{D_{n}=k\right\}=d_{k} .
$$

In a companion paper [6], we have established that the classes of 2-connected, connected or all outerplanar graphs, as well as the corresponding classes of seriesparallel graphs have a degree distribution. We describe briefly the degree distribution in the outerplanar case, which is the simplest one, and refer to [6] for the other

\footnotetext{
${ }^{1}$ Alternatively we can define $D_{n}$ as the degree of the vertex with label 1.
} 
cases. Let $d_{k}$ be defined as before for outerplanar graphs, let

$$
D(x)=\frac{1+x-\sqrt{1-6 x+x^{2}}}{4},
$$

and let

$$
g(x, w)=x w+\frac{x w^{2}}{2} \frac{2 D(x)-x}{1-(2 D(x)-x) w} .
$$

The function $D(x)$ is a minor modification of function $A(x)$ in $(2.1)$, and $g(x, w)$ is the generating function of rooted of 2-connected outerplanar graphs, where $w$ marks the degree of the root.

Then we have

$$
\sum_{k \geq 0} d_{k} w^{k}=\left.\rho \cdot \frac{\partial}{\partial x} e^{g(x, w)}\right|_{x=\tau},
$$

where $\rho$ and $\tau$ are constants defined analytically and having approximate values $\rho \approx 0.1366$ and $\tau \approx 0.1708$. A plot of the distribution is given in Figure 1. For comparison, we have added also a plot of the distribution for 2-connected outerplanar graphs, whose probability generating function can be computed directly from $g(x, w)$.

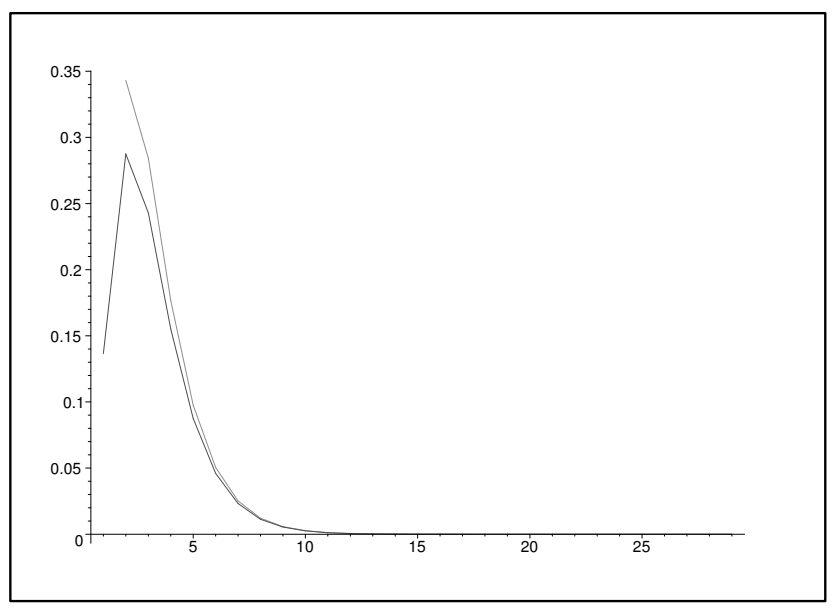

Figure 1. Degree distribution for outerplanar graphs (bottom graph) and 2-connected outerplanar graphs (top graph).

In what follows we consider the random variables $X_{n}^{(k)}$ that count the number of vertices of degree $k$ in a random graph with $n$ vertices of the above mentioned types. It is clear that $D_{n}$ and $X_{n}^{(k)}$ are closely related, for example we have

$$
\operatorname{Pr}\left\{D_{n}=k\right\}=\frac{\mathbf{E} X_{n}^{(k)}}{n} .
$$

This means that a degree distribution $\left\{d_{k}\right\}$ exists if and only if $\mathbf{E} X_{n}^{(k)} \sim d_{k} n$ (as $n \rightarrow \infty$ ), for all $k \geq 0$.

The main goal of this paper is to obtain more precise information on the random variables $X_{n}^{(k)}$. In particular we are interested in probabilistic limit theorems and tail estimates. 
In order to state our results concisely, we say that a sequence of random variables $Y_{n}$ satisfies a central limit theorem with linear expected value and variance if there exist constants $\mu \geq 0$ and $\sigma^{2} \geq 0$ such that

$$
\mathbf{E} Y_{n}=\mu n+O(1), \quad \operatorname{Var} X_{n}=\sigma^{2} n+O(1),
$$

and

$$
\frac{Y_{n}-\mathbf{E} Y_{n}}{\sqrt{n}} \rightarrow N\left(0, \sigma^{2}\right),
$$

where $N\left(0, \sigma^{2}\right)$ denotes the normal distribution with zero mean and variance $\sigma^{2}$. (The reason why we do not divide by $\sigma^{2}$ is that we also want to cover the eventual case of zero variance. Note that $N(0,0)$ is the delta distribution concentrated at 0 .)

Furthermore we say that $Y_{n}$ has quadratic exponential tail estimates if there exist positive constants $c_{1}, c_{2}, c_{3}$ such that

$$
\operatorname{Pr}\left\{\left|Y_{n}-\mathbf{E} Y_{n}\right| \geq \varepsilon \sqrt{n}\right\} \leq c_{1} e^{-c_{2} \epsilon^{2}}
$$

uniformly for $0 \leq \varepsilon \leq c_{3} \sqrt{n}$. Note that (1.1) is equivalent to

$$
\operatorname{Pr}\left\{\left|Y_{n}-\mathbf{E} Y_{n}\right| \geq \varepsilon n\right\} \leq c_{1} e^{-c_{2} \epsilon^{2} n}
$$

for $0 \leq \varepsilon \leq c_{3}$.

Our main results are the following.

Theorem 1.1. For $k \geq 1$, let $X_{n}^{(k)}$ denote the number of vertices of degree $k$ in random 2-connected, connected or unrestricted labelled outerplanar graphs with $n$ vertices. Then $X_{n}^{(k)}$ satisfies a central limit theorem with linear expected value and variance and has quadratic exponential tail estimates.

Theorem 1.2. For $k \geq 1$, let $X_{n}^{(k)}$ denote the number of vertices of degree $k$ in random 2-connected, connected or unrestricted labelled series-parallel graphs with $n$ vertices. Then $X_{n}^{(k)}$ satisfies a central limit theorem with linear expected value and variance and has quadratic exponential tail estimates.

Theorem 1.3. For $k=1,2$, let $X_{n}^{(k)}$ denote the number of vertices of degree $k$ in random 2-connected, connected or unrestricted labelled planar graphs with $n$ vertices. Then $X_{n}^{(k)}$ satisfies a central limit theorem with linear expected value and variance and has quadratic exponential tail estimates.

Strictly speaking we should exclude the case $k=1$ for 2-connected graphs in the previous theorems, since $X_{n}^{(1)}$ is identically zero in this case. As we discuss later, the case $k=1$ is already proved in [10] for planar graphs, and the proof also works in the remaining cases.

Further, the case $k=0$ is excluded in Theorems 1.1-1.3 since the expected number of nodes of degree 0 , that is, one-vertex components, is bounded.

We note that our methods provide even more general results than stated above. For example we also get a multivariate normal limit law for the random vector $\left(X_{n}^{(1)}, \ldots, X_{n}^{(k)}\right)$. Further let us mention that 2-connected outerplanar graphs are very close to polygon dissections. In particular, we also get a central limit theorem for the number of vertices of degree $k$ in random dissections.

We have shown in [6] that random planar graphs also have a degree distribution, and it is only natural to ask whether Theorem 1.3 can be extended to degrees greater than 2. This is a tantalizing open problem and it appears to us that the 
tools we have presently at our disposal for analyzing planar graphs (like those in [10], where the problem of counting planar graphs was solved) are not sufficient. We cannot even prove a central limit theorem for vertices of degree $k$ in 3-connected planar graphs, which would be the first natural step in this problem. However, we believe that a central limit theorem holds for planar graphs too.

The structure of the paper is as follows. We first present relations for generating functions in several variables that count outerplanar and series-parallel graphs with respect to their total number of vertices and with respect to their number of vertices of given degree (Sections 2 and 3). In particular we set up systems of equations for the corresponding generating functions. This is also done for planar graphs and degree two in Section 4.

Sections 5 and 6 are devoted to the discussion of two analytic tools that we use to prove Theorems 1.1-1.3. First we discuss several transfer principles of singularities that are needed in the proofs. Secondly, we collect results that provide the singular structure of solutions of systems of functional equations. It turns out that the singularities are (usually) of square-root type. With the help of the transfer lemma of Flajolet and Odlyzko [8], this singular behaviour transfers into an asymptotic relation for the probability generating function $\mathbf{E} u^{X_{n}^{(k)}}$, that directly proves asymptotic normality and provides tail estimates, too.

The proof of Theorems 1.1-1.3 are then given in the last three sections. The method of proof is the following. First, in each of the three cases (outerplanar, series-parallel and planar) we introduce generating functions $B_{j}^{\bullet}$ of 2 -connected graphs rooted at a vertex, where the root has degree $j$. In each case we show that the $B_{j}^{\bullet}$ can be expressed in terms of other generating functions satisfying a system of functional equations. For outerplanar graphs, this is done through polygon dissections; for series-parallel graphs, we use series-parallel networks; and for planar graphs and degree two, we use a direct argument involving the seriesparallel reduction of a 2-connected planar graph. Applying the tools from Sections 5 and 6 we get central limit theorems for 2-connected graphs.

Then we introduce analogous generating functions $C_{j}^{\bullet}$ for connected graphs, again rooted at a vertex and where the root has degree $j$. There is a universal relation between the $C_{j}^{\bullet}$ and the $B_{j}^{\bullet}$, which reflects the decomposition of a connected graph into 2-connected components (or blocks). This is the content of Lemma 2.6. For a given family of graphs $\mathcal{G}$, Lemma 2.6 holds whenever a connected graph is in $\mathcal{G}$ if and only if its 2-connected components are in $\mathcal{G}$; this is clearly the case for the three families we consider in this paper. Finally, in order to go from connected graphs to arbitrary graphs, we use the exponential formula (2.6), which reflects the fact that a graph is in one of our classes if and only if its connected components are also in the class. Again using the tools developed in Sections 5 and 6 we get central limit theorems and tail estimates.

We wish to point out that the results on transfer of singularities in bivariate generating functions from Section 5, that we have developed for the needs of the present paper, have a wide range of applications and can be useful in other problems of graph enumeration where one has to consider at the same time graphs rooted at a vertex and graphs rooted at an edge.

The results from Section 6 are refined versions of the main findings in [5] and shall give more light on the relation between systems of equations of generating 
functions and central limit theorems. It appears that this approach has far reaching applications.

\section{Generating Functions for Outerplanar Graphs}

There is an intimate relation between dissections (that we discuss first) and outerplanar graphs. However, the derivation for the generating functions that involve the number of vertices of given degree are easier to state and derive within the framework of dissections. The corresponding generating functions for outerplanar graphs can then be derived from the previous ones.

Recall that an outerplanar graph is a planar graph (embedded in the plane) where all vertices are on the infinite face.

2.1. Dissections. A dissection is a convex polygon together with a set of noncrossing diagonals. In our context we will further assume that one edge of the polygon is rooted (or marked), see Figure 2. Alternatively we can interpret a dissection as an outerplanar graph with a rooted edge on the infinite face.

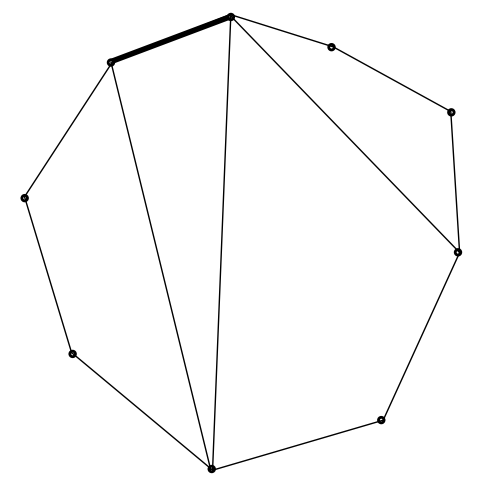

Figure 2. Dissection of a convex polygon.

Let $\mathcal{A}$ denote the set of dissections with at least 3 vertices and let $a_{n}, n \geq 1$, be the number of dissections with $n+2$ vertices, that is, the vertices of the marked edge are not counted. Further, let

$$
A(x)=\sum_{n \geq 1} a_{n} x^{n}
$$

denote the corresponding generating function.

We first state (and prove) well known properties of $A(x)$ and the numbers $a_{n}$ (compare with [7]). The reason why we present a detailed proof is that we will need the ideas of the combinatorial constructions in the subsequent generalizations, where we take degrees of vertices into account.

Lemma 2.1. The generating function $A(x)$ satisfies the functional equation

$$
A(x)=x(1+A(x))^{2}+x(1+A(x)) A(x)
$$

and has an explicit representation of the form

$$
A(x)=\frac{1-3 x-\sqrt{1-6 x+x^{2}}}{4 x} .
$$


Furthermore, the numbers $a_{n}$ are explicitly given by

$$
a_{n}=\frac{1}{n} \sum_{\ell=0}^{n-1}\left(\begin{array}{l}
n \\
\ell
\end{array}\right)\left(\begin{array}{c}
n \\
\ell+1
\end{array}\right) 2^{\ell} .
$$

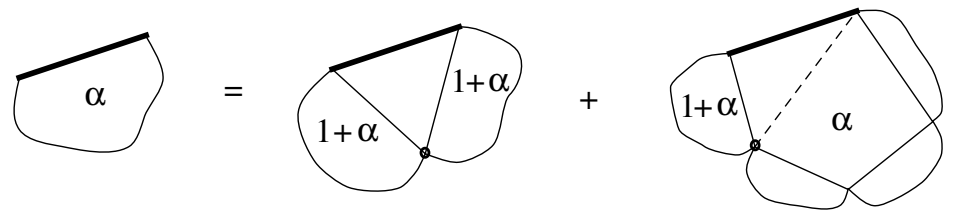

FiguRE 3. Recursive decomposition of dissections.

Proof. We first note that dissections have an easy recursive description (see with Figure 3). Every dissection $\alpha$ has a unique face $f$ that contains the root edge. Suppose first that $f$ contains exactly three vertices, that is, $f$ is a triangle, and denote the three edges of $f$ by $e=\left(v_{1}, v_{2}\right)$, the root edge, and by $e_{1}=\left(v_{2}, v_{3}\right)$ and $e_{2}=\left(v_{3}, v_{1}\right)$. (We always label the vertices in counter-clockwise order.) We can then decompose $\alpha$ into three parts. We cut $\alpha$ at the three vertices $v_{1}, v_{2}, v_{3}$ of $f$ and get first the root edge $e$, then a part $\alpha_{1}$ of $\alpha$ that contains $e_{1}$, and finally a third part $\alpha_{2}$ that contains $e_{2}$. Obviously $\alpha_{1}$ and $\alpha_{2}$ are again connected planar graphs, where $e_{1}$ and $e_{2}$ can be viewed as rooted edges. Since all vertices of $\alpha$ are on the infinite face the same holds for $\alpha_{1}$ and $\alpha_{2}$, however, $\alpha_{1}$ and $\alpha_{2}$ might consists just of $e_{1}$ or $e_{2}$. Thus, $\alpha_{1}$ and $\alpha_{2}$ are either just one (rooted) edge or again a dissection. By counting the number of vertices in the above way this case corresponds to the generating function $x(1+A(x))^{2}$.

If $f$ contains more than three edges then we first cut $f$ into two pieces $f_{1}$ and $f_{2}$, where $f_{1}$ consists of the root edge $e=\left(v_{1}, v_{2}\right)$, the adjacent edge $e_{1}=\left(v_{2}, v_{3}\right)$, and a new edge $e_{n e w}=\left(v_{3}, v_{1}\right)$. We again cut $\alpha$ at the vertices $v_{1}, v_{2}, v_{3}$ and get, first, the root edge $e$, then a part $\alpha_{1}$ of $\alpha$ that contains $e_{1}$ and a third part $\tilde{\alpha}_{2}$ that contains the new edge $e_{\text {new }}$. As above $\alpha_{1}$ is either $e_{1}$ or is a dissection rooted at $e_{1}$. Since $f$ has more than three edges, $\tilde{\alpha}_{2}$ has at least three vertices. Hence, we can consider $\tilde{\alpha}_{2}$ as a dissection rooted at $e_{n e w}$. Similarly to the above, this case corresponds to the generating function $x(1+A(x)) A(x)$.

It is now an easy application of Lagrange's inversion formula to obtain an explicit representation for $a_{n}$.

Remark. It is shown in [7] that the radius of convergence of $A(x)$ is given by $\rho_{1}=3-2 \sqrt{2}$ and the asymptotic expansion of $a_{n}$ is given by

$$
a_{n}=c n^{-\frac{3}{2}} \rho_{1}^{-n}\left(1+O\left(\frac{1}{n}\right)\right),
$$

where the constant $c$ is given by

$$
c=\frac{1}{4 \sqrt{\pi}} \sqrt{99 \sqrt{2}-140} .
$$


Next we want to take into account the number of vertices of a given degree. For this purpose we fix some $k \geq 2$ and extend our generating function counting procedure, using variables $x, z_{1}, z_{2}, \ldots, z_{k}, z_{\infty}$, where the variable $z_{\ell}, 1 \leq \ell \leq k$, marks vertices of degree $\ell$, and $z_{\infty}$ marks vertices of degree greater than $k$. Furthermore, we consider the degrees $i, j$ of the vertices $v_{1}$ and $v_{2}$ of the root edge $e=\left(v_{1}, v_{2}\right)$. More precisely, if

$$
a_{i, j ; n, n_{1}, n_{2}, \ldots, n_{k}, n_{\infty}}
$$

is the number of dissections with $2+n_{1}+n_{2}+\cdots+n_{k}+n_{\infty} \geq 3$ vertices such that the two vertices $v_{1}, v_{2}$ of the marked edge $e=\left(v_{1}, v_{2}\right)$ have degrees $d\left(v_{1}\right)=i$ and $d\left(v_{2}\right)=j$, and that for $1 \leq \ell \leq k$ there are $n_{\ell}$ vertices $v \neq v_{1}, v_{2}$, with $d(v)=\ell$, and there are $n_{\infty}$ vertices $v \neq v_{1}, v_{2}$, with $d(v)>k$. The corresponding generating functions are then defined by

$$
A_{i, j}\left(x, z_{1}, z_{2}, \ldots, z_{k}, z_{\infty}\right)=\sum_{n, n_{1}, \ldots, n_{k}, n_{\infty}} a_{i, j ; n, n_{1}, n_{2}, \ldots, n_{k}, n_{\infty}} x^{n} z_{1}^{n_{1}} \cdots z_{k}^{n_{k}} z_{\infty}^{n_{\infty}} .
$$

Similarly we define $A_{i, \infty}, A_{\infty, j}$ and $A_{\infty, \infty}$ if one (or both) of the vertices of the root edge have degree(s) greater than $k$.

Note that $z_{1}$ is not necessary since there are no vertices of degree one in dissections. However, we use $z_{1}$ for later purposes. Further observe that $A_{i j}\left(z_{1}, z_{2}, \ldots, z_{k}, z_{\infty}\right)=A_{j i}\left(z_{1}, z_{2}, \ldots, z_{k}, z_{\infty}\right)$. Thus, it is sufficient to consider $A_{i j}$ for $i \leq j$.

In order to state the following lemma in a more compact form we use the convention that $\infty$ means $>k$, and $\infty-1$ means $>k-1$. In particular we set $\ell+\infty=\infty$ for all positive integers $\ell$.

The concept of a strongly connected positive system of equations is explained in detail in Section 6. Informally it says that on the right hand side of the equations there are no minus signs and that it is impossible to solve a subsystem before solving the whole system.

Lemma 2.2. The generating functions $A_{i j}=A_{j i}=A_{i j}\left(x, z_{1}, z_{2}, \ldots, z_{k}, z_{\infty}\right)$, $i, j \in\{2,3, \ldots, k, \infty\}$, satisfy the following strongly connected positive system of equations:

$$
\begin{aligned}
A_{i j} & =x \sum_{\ell_{1}+\ell_{2} \leq k} z_{\ell_{1}+\ell_{2}} A_{i-1, \ell_{1}} A_{j-1, \ell_{2}}+z_{\infty}\left(\sum_{\ell_{1}+\ell_{2}>k} A_{i-1, \ell_{1}} A_{j-1, \ell_{2}}\right) \\
& +x \sum_{\ell_{1}+\ell_{2} \leq k+1} z_{\ell_{1}+\ell_{2}-1} A_{i-1, \ell_{1}} A_{j, \ell_{2}}+x z_{\infty}\left(x \sum_{\ell_{1}+\ell_{2}>k+1} A_{i-1, \ell_{1}} A_{j, \ell_{2}}\right) .
\end{aligned}
$$

One has to be careful in writing down the equations explicitly. For example we have

$$
\begin{aligned}
A_{i, \infty} & =x \sum_{\ell_{1}+\ell_{2} \leq k} z_{\ell_{1}+\ell_{2}} A_{i-1, \ell_{1}}\left(A_{k, \ell_{2}}+A_{\infty, \ell_{2}}\right)+x z_{\infty}\left(\sum_{\ell_{1}+\ell_{2}>k} A_{i-1, \ell_{1}}\left(A_{k, \ell_{2}}+A_{\infty, \ell_{2}}\right)\right) \\
& +x \sum_{\ell_{1}+\ell_{2} \leq k+1} z_{\ell_{1}+\ell_{2}-1} A_{i-1, \ell_{1}} A_{\infty, \ell_{2}}+x z_{\infty}\left(\sum_{\ell_{1}+\ell_{2}>k+1} A_{i-1, \ell_{1}} A_{\infty, \ell_{2}}\right) .
\end{aligned}
$$


As an illustration, for $k=3$ we have the following system:

$$
\begin{aligned}
A_{22} & =x z_{2} \\
& +x z_{2} A_{22}+x z_{3} A_{23}+x z_{\infty} A_{2 \infty} \\
A_{23} & =x z_{3} A_{22}+x z_{\infty}\left(A_{23}+A_{2 \infty}\right) \\
& =x z_{2} A_{23}+x z_{3} A_{33}+x z_{\infty} A_{3 \infty} \\
A_{2 \infty} & =x z_{3} A_{23}+x z_{\infty}\left(A_{33}+A_{3 \infty}\right)+x z_{\infty}\left(A_{2 \infty}+A_{3 \infty}+A_{\infty, \infty}\right) \\
& +x z_{2} A_{2 \infty}+x z_{3} A_{3 \infty}+x z_{\infty} A_{\infty, \infty} \\
A_{33} & =x z_{\infty}\left(A_{22}+A_{23}+A_{2 \infty}\right)^{2} \\
& +x z_{\infty}\left(A_{22}+A_{23}+A_{2 \infty}\right)\left(A_{23}+A_{33}+A_{3 \infty}\right), \\
A_{3 \infty} & =x z_{\infty}\left(A_{23}+A_{33}+A_{3 \infty}\right)\left(A_{2 \infty}+A_{3 \infty}+A_{\infty, \infty}\right) \\
& +x z_{\infty}\left(A_{22}+A_{23}+A_{2 \infty}\right)\left(A_{2 \infty}+A_{3 \infty}+A_{\infty, \infty}\right) \\
A_{\infty, \infty} & =x z_{\infty}\left(A_{23}+A_{33}+A_{3 \infty}+A_{2 \infty}+A_{3 \infty}+A_{\infty, \infty}\right)^{2} \\
& +x z_{\infty}\left(A_{23}+A_{33}+A_{3 \infty}+A_{2 \infty}+A_{3 \infty}+A_{\infty, \infty}\right)\left(A_{2 \infty}+A_{3 \infty}+A_{\infty, \infty}\right) .
\end{aligned}
$$

Proof. The idea is to have a more detailed look at the proof of the recursive structure of $\mathcal{A}$ as described in the proof of Lemma 2.1.

We only discuss the recurrence for $A_{i j}$ for finite $i, j$. If $i=\infty$ or $j=\infty$ similar considerations apply. The root edge will be denoted by $e=\left(v_{1}, v_{2}\right)$. We assume that $v_{1}$ has degree $j$ and $v_{2}$ has degree $i$. Again we have to distinguish between the case where the face $f$ containing the root edge $e$ has exactly three edged, and the case where it has more than three edged.

In the first case we cut a dissection $\alpha$ at the three vertices $v_{1}, v_{2}, v_{3}$ of $f$ and get the root edge $e$, and two dissections $\alpha_{1}$ and $\alpha_{2}$ that are rooted at $e_{1}=\left(v_{2}, v_{3}\right)$ and $e_{2}=\left(v_{3}, v_{1}\right)$. After the cut, $\alpha_{1}$ has degree $i-1$ at $v_{2}$, and $\alpha_{2}$ has degree $j-1$ at $v_{1}$. Furthermore, the total degree of the common vertex $v_{3}$ is just the sum of the degrees coming from $\alpha_{1}$ and $\alpha_{2}$. Hence, if the degree of $v_{3}$ is smaller or equal than $k$, then this situation corresponds to the generating function

$$
x \sum_{\ell_{1}+\ell_{2} \leq k} z_{\ell_{1}+\ell_{2}} A_{i-1, \ell_{1}} A_{j-1, \ell_{2}} .
$$

Since all possible cases for $\alpha_{1}$ are encoded in $A_{i-1}=A_{i-1,2}+\cdots+A_{i-1, k}+A_{i-1, \infty}$, and all cases for $\alpha_{2}$ are encoded in $A_{j-1}$, it follows that all situations where the total degree of $v_{3}$ is greater than $k$ are given by the generating function

$$
x z_{\infty}\left(\sum_{\ell_{1}+\ell_{2}>k} A_{i-1, \ell_{1}} A_{j-1, \ell_{2}}\right) .
$$

Similarly we argue in the case where $f$ contains more than three edges. After cutting $f$ into two pieces $f_{1}$ and $f_{2}$ with a new edge $e_{n e w}=\left(v_{3}, v_{1}\right)$, and cutting $\alpha$ at the vertices $v_{1}, v_{2}, v_{3}$, we get again the root edge $e$ and two dissections $\alpha_{1}$ and $\alpha_{2}$ that are rooted at $e_{1}=\left(v_{2}, v_{3}\right)$ and at the new edge $e_{n e w}=\left(v_{3}, v_{1}\right)$. After the cut, $\alpha_{1}$ has degree $i-1$ at $v_{2}$ and $\alpha_{2}$ has degree $j$ at $v_{1}$, since the new edge $e_{n e w}$ has to be taken into account. The total degree of the common vertex $v_{3}$ is the sum of the degrees coming from $\alpha_{1}$ and $\alpha_{2}$ minus 1 , since the new edge $e_{n e w}$ is used in the construction of $\alpha_{2}$. As above these observations translate into the generating 
functions

$$
x \sum_{\ell_{1}+\ell_{2} \leq k+1} z_{\ell_{1}+\ell_{2}-1} A_{i-1, \ell_{1}} A_{j, \ell_{2}}
$$

and

$$
x z_{\infty}\left(\sum_{\ell_{1}+\ell_{2}>k+1} A_{i-1, \ell_{1}} A_{j, \ell_{2}}\right) .
$$

Finally, by using the definition of $A_{i}$ and $A_{\infty}$, it follows that the above system of equations is a positive one, that is, all coefficients on the right hand side are non-negative. Further, it is easy to check that the corresponding dependency graph is strongly connected, which means that no subsystem can be solved before the whole system is solved.

2.2. 2-Connected Outerplanar Graphs. We consider now 2-connected outerplanar graphs where the vertices are labelled (see Figure 4). There is an obvious relation between dissections and 2-connected outerplanar graphs.

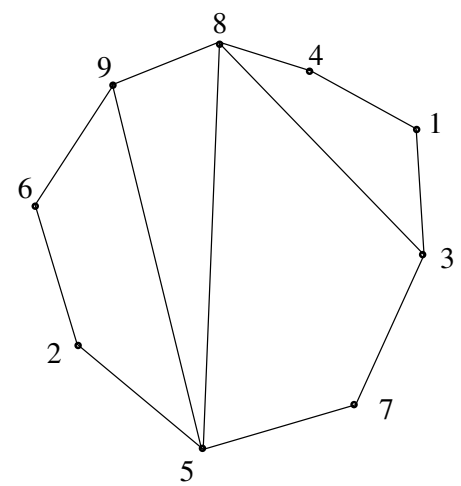

FigurE 4. A 2-Connected outerplanar graph.

Lemma 2.3. Let $b_{n}, n \geq 2$, be the number of 2-connected outerplanar labelled graphs. Then the exponential generating function

$$
B(x)=\sum_{n \geq 2} b_{n} \frac{x^{n}}{n !}
$$

satisfies

$$
B^{\prime}(x)=x+\frac{1}{2} x A(x),
$$

where $A(x)$ is the generating function of dissections, given by (2.1).

Proof. There is exactly one 2-connected outerplanar graph with two vertices, namely a single edge. If $n \geq 3$ then we have

$$
b_{n}=\frac{(n-1) !}{2} a_{n-2}
$$

First, it is clear that $b_{n}$ can be also considered as the number of 2-connected outerplanar graphs with $n$ vertices, where one vertex is marked (or rooted) and the 
remaining $n-1$ vertices are labelled by $1,2, \ldots, n-1$. We just have to identify the vertex with label $n$ with the marked vertex. Next consider a dissection with $n$ vertices. There are $a_{n-2}$ dissections of that kind. We mark the vertex $v_{1}$ of the root edge $e=\left(v_{1}, v_{2}\right)$ (where the vertices are numbered counter clockwise). Then there are exactly $(n-1)$ ! ways to label the remaining $n-1$ vertices by $1,2, \ldots, n-1$. Finally since the direction of the outer circle is irrelevant, we have to divide the resulting number $(n-1) ! a_{n-2}$ by 2 to get back $b_{n}$.

Remark. With help of Lemma 2.1 we can also derive an explicit formula for $B(x)$ :

$B(x)=\frac{x}{8}+\frac{5}{16} x^{2}-\frac{1}{32}(-6+2 x) \sqrt{1-6 x+x^{2}}+\frac{1}{2} \log \left(-3+x+\sqrt{1-6 x+x^{2}}\right)$

By looking at the proof of Lemma 2.3, the derivative

$$
B^{\prime}(x)=\sum_{n \geq 2} b_{n} \frac{x^{n-1}}{(n-1) !}=\sum_{n \geq 1} b_{n+1} \frac{x^{n}}{n !}
$$

can also be interpreted as the exponential generating function $B^{\bullet}(x)$ of 2 -connected outerplanar graphs, where one vertex is marked and is not counted. We make heavy use of this interpretation in the sequel of the paper. In particular, we set $b_{n}^{\bullet}=b_{n+1}$.

Next we set

$$
B_{j}^{\bullet}\left(x, z_{1}, z_{2}, \ldots, z_{k}, z_{\infty}\right)=\sum_{n, n_{1}, \ldots, n_{k}, n_{\infty}} b_{j ; n, n_{1}, \ldots, n_{k}, n_{\infty}}^{\bullet} \frac{x^{n} z_{1}^{n_{1}} \cdots z_{k}^{n_{k}} z_{\infty}^{n_{\infty}}}{n !},
$$

where $b_{j ; n, n_{1}, \ldots, n_{k}, n_{\infty}}^{\bullet}$ is the number of 2-connected outerplanar graphs with $1+n=$ $1+n_{1}+\cdots+n_{k}+n_{\infty}$ vertices, where one vertex of degree $j$ is marked and the remaining $n$ vertices are labelled by $1,2, \ldots, n$ and where $n_{\ell}$ vertices have degree $\ell, 1 \leq \ell \leq k$, and $n_{\infty}$ vertices have degree greater than $k$.

Lemma 2.4. Let $A_{i j}=A_{j i}=A_{i j}\left(x, z_{1}, z_{2}, \ldots, z_{k}, z_{\infty}\right), i, j \in\{1,2, \ldots, k, \infty\}$ be defined by (2.2). Then the functions $B_{j}=B_{j}^{\bullet}\left(x, z_{1}, z_{2}, \ldots, z_{k}, z_{\infty}\right), j \in$ $\{1,2, \ldots, k, \infty\}$, are given by

$$
\begin{aligned}
B_{1}^{\bullet} & =x z_{1} \\
B_{j}^{\bullet} & =\frac{1}{2} \sum_{i=1}^{k} x z_{i} A_{i j}+\frac{1}{2} x z_{\infty} A_{j \infty} \\
B_{\infty}^{\bullet} & =\frac{1}{2} \sum_{i=1}^{k} x z_{i} A_{j \infty}+\frac{1}{2} x z_{\infty} A_{\infty, \infty} .
\end{aligned}
$$

Proof. The proof is immediate by repeating the arguments of Lemma 2.3 and by taking care of the vertex degrees.

Now let

$$
B_{d=k}(x, u)=\sum_{n, \nu} b_{n, \nu}^{(k)} \frac{x^{n}}{n !} u^{\nu}
$$

denote the exponential generating function for the the numbers $b_{n, \nu}^{(k)}$ of 2-connected outerplanar labelled graphs with $n$ vertices, where $\nu$ vertices have degree $k$. Then 
we have

$$
\frac{\partial B_{d=k}(x, u)}{\partial x}=\sum_{j=1}^{k-1} B_{j}^{\bullet}(x, 1, \ldots, 1, u, 1)+u B_{k}^{\bullet}(x, 1, \ldots, 1, u, 1)+B_{\infty}^{\bullet}(x, 1, \ldots, 1, u, 1) .
$$

Since $B(0, u)=0$ this equation completely determines $B_{d=k}(x, u)$.

2.3. Connected Outerplanar Graphs. There is a general relation between rooted 2-connected graphs and rooted connected graphs. We now state it for outerplanar graphs but the following lemma is also valid, for example, for series parallel graphs or for general planar graphs $[10,3]$.

Lemma 2.5. Let $B^{\bullet}(x)$ be the exponential generating function of 2-connected rooted outerplanar graphs and $C^{\bullet}(x)$ the corresponding exponential generating function of connected rooted outerplanar graphs. Then we have

$$
C^{\bullet}(x)=e^{B^{\bullet}\left(x C^{\bullet}(x)\right)} .
$$

Proof. The right hand side

$$
e^{B^{\bullet}\left(x C^{\bullet}(x)\right)}=\sum_{k=0}^{\infty} \frac{1}{k !} B^{\bullet}\left(x C^{\bullet}(x)\right)^{k}
$$

of the equation (2.4) can be interpreted as the exponential generating function of a finite set of rooted 2-connect graphs, where the root vertices are identified to form a new connected rooted graph, and every vertex different from the root is replaced by a rooted connected graph (see Figure 5).

It is now easy to show that every rooted connected planar graph $G$ can be decomposed uniquely in the above way. Let $v_{\text {root }}$ denote the root vertex of a connected graph. If we delete an arbitrary vertex $v \neq v_{\text {root }}$, then the graph decomposes into $j \geq 1$ components $G_{1}, G_{2}, \ldots G_{j}$, where we assume that the root $v_{\text {root }}$ is contained in $G_{1}$. We now reduce $G$ to a graph $G^{\prime}$ by deleting $\left(G_{2} \cup \cdots \cup G_{j}\right) \backslash\{v\}$ from $G$. By repeating this procedure we end up with a graph $\tilde{G}$. Finally we delete the root $v_{\text {root }}$ and obtain $k \geq 1$ connected graphs $\tilde{G}_{1}, \ldots, \tilde{G}_{k}$. Let $B_{i}, 1 \leq i \leq j$, denote the graph $\tilde{G}_{i}$ together with $v_{\text {root }}$ and all edges from $v_{\text {root }}$ to $\tilde{G}_{i}$. Then $B_{i}, 1 \leq i \leq j$, is a 2 -connected planar graph that is rooted at $v_{\text {root }}$, and $\tilde{G}$ is obtained by identifying all the root vertices. This gives the required decomposition.

Remark. We know from [3] that $C^{\bullet}(x)$ has radius of convergence $\rho=0.1366 \cdots$ and that $\rho C^{\bullet}(\rho)<\rho_{1}=3-2 \sqrt{2}$; this implies that the singularity of $B^{\bullet}(x)$ is irrelevant for the analysis of the singular behaviour of $C^{\bullet}(x)$ for $x \rightarrow \rho$.

Next we discuss the generating functions

$$
C_{j}^{\bullet}\left(x, z_{1}, z_{2}, \ldots, z_{k}, z_{\infty}\right)=\sum_{n, n_{1}, \ldots, n_{k}, n_{\infty}} c_{j ; n ; n_{1}, \ldots, n_{k}, n_{\infty}}^{\bullet} z_{1}^{n_{1}} \cdots z_{k}^{n_{k}} z_{\infty}^{n_{\infty}} \frac{x^{n}}{n !},
$$

$j \in\{1,2, \ldots, k, \infty\}$, where $c_{j ; n ; n_{1}, \ldots, n_{k}, n_{\infty}}^{\bullet}$ is the number of connected outerplanar graphs with $1+n=1+n_{1}+\cdots+n_{k}+n_{\infty}$ vertices, where one vertex of degree $j$ is marked $^{2}$ and the remaining $n$ vertices are labelled by $1,2, \ldots, n$ and where $n_{\ell}$

\footnotetext{
${ }^{2}$ If $j=\infty$ this has to be interpreted as a vertex of degree $>k$.
} 


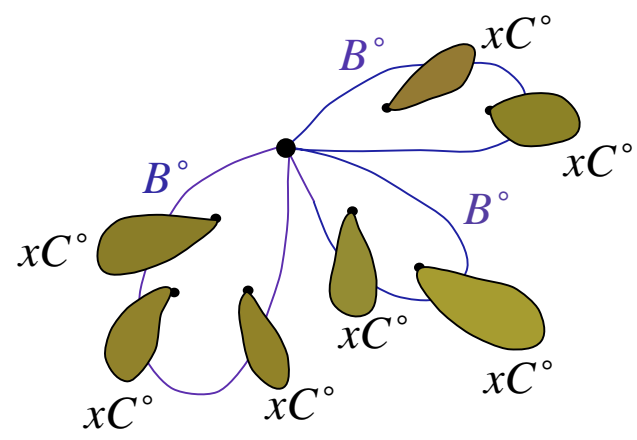

FiguRE 5. Connection between 2-connected and connected planar graphs.

of these $n$ vertices have degree $\ell, 1 \leq \ell \leq k$, and $n_{\infty}$ of these vertices have degree greater than $k$. For convenience, we also define

$$
C_{0}^{\bullet}\left(x, z_{1}, z_{2}, \ldots, z_{k}, z_{\infty}\right)=1,
$$

which corresponds to the case of a graph with a single rooted vertex.

Lemma 2.6. Let $W_{j}=W_{j}\left(z_{1}, \ldots, z_{k}, z_{\infty}, C_{1}^{\bullet}, \ldots, C_{k}^{\bullet}, C_{\infty}^{\bullet}\right), j \in\{1,2, \ldots, k, \infty\}$, be defined by

$$
\begin{aligned}
W_{j} & =\sum_{i=0}^{k-j} z_{i+j} C_{i}^{\bullet}\left(x, z_{1}, \ldots, z_{k}, z_{\infty}\right) \\
& +z_{\infty}\left(\sum_{i=k-j+1}^{k} C_{i}^{\bullet}\left(x, z_{1}, \ldots, z_{k}, z_{\infty}\right)+C_{\infty}^{\bullet}\left(x, z_{1}, \ldots, z_{k}, z_{\infty}\right)\right), \\
& (1 \leq j \leq k), \\
W_{\infty} & =z_{\infty}\left(\sum_{i=0}^{k} C_{i}^{\bullet}\left(x, z_{1}, \ldots, z_{k}, z_{\infty}\right)+C_{\infty}^{\bullet}\left(x, z_{1}, \ldots, z_{k}, z_{\infty}\right)\right) .
\end{aligned}
$$

Then the functions $C_{1}^{\bullet}, \ldots, C_{k}^{\bullet}, C_{\infty}^{\bullet}$ satisfy the system of equations

$$
\begin{aligned}
C_{j}^{\bullet}\left(x, z_{1}, \ldots, z_{k}, z_{\infty}\right) & =\sum_{\substack{\ell_{1}+2 \ell_{2}+3 \ell_{3}+\cdots j \ell_{j}=j \\
(1 \leq j \leq k),}} \prod_{r=1}^{j} \frac{B_{r}^{\bullet}\left(x, W_{1}, \ldots, W_{k}, W_{\infty}\right)^{\ell_{r}}}{\ell_{r} !} \\
C_{\infty}^{\bullet}\left(x, z_{1}, \ldots, z_{k}, z_{\infty}\right) & =\exp \left(\sum_{j=1}^{k} B_{j}^{\bullet}\left(x, W_{1}, \ldots, W_{k}, W_{\infty}\right)+B_{\infty}^{\bullet}\left(x, W_{1}, \ldots, W_{k}, W_{\infty}\right)\right) \\
& -1-\sum_{1 \leq \ell_{1}+2 \ell_{2}+3 \ell_{3}+\cdots k \ell_{k} \leq k} \prod_{r=1}^{k} \frac{B_{r}^{\bullet}\left(x, W_{1}, \ldots, W_{k}, W_{\infty}\right)^{\ell_{r}}}{\ell_{r} !} .
\end{aligned}
$$


Proof. The proof is a refined version of the proof of Lemma 2.5, which reflects the decomposition of a rooted connected rooted graphs into a finite set of rooted 2connected graphs where every vertex (different from the root) is substituted by a rooted connected graph. Functions $W_{j}$ serve the purpose of marking (recursively) the degree of the vertices in the 2-connected blocks which are substituted by other graphs. If we look at the definition of $W_{j}$, the summation means that we are substituting for a vertex of degree $i$, but since originally the vertex had degree $j$, we are creating a new vertex of degree $i+j$, which is marked accordingly by $z_{i+j}$. The same remark applies to $W_{\infty}$.

Finally let

$$
C_{d=k}(x, u)=\sum_{n, \nu} c_{n, \nu}^{(k)} \frac{x^{n}}{n !} u^{\nu}
$$

denote exponential generating function for the the numbers $c_{n, \ell}^{(k)}$ of connected outerplanar vertex labelled graphs with $n$ vertices, where $\nu$ vertices have degree $k$. Then we have

$\frac{\partial C_{d=k}(x, u)}{\partial x}=\sum_{j=1}^{k-1} C_{j}^{\bullet}(x, 1, \ldots, 1, u, 1)+u C_{k}^{\bullet}(x, 1, \ldots, 1, u, 1)+C_{\infty}^{\bullet}(x, 1, \ldots, 1, u, 1)$.

Since $C(0, u)=0$ this equation completely determines $C_{d=k}(x, u)$.

2.4. All Outerplanar Graphs. It is then also possible to consider non-connected outerplanar graphs. Clearly the generating function $G(x)$ and the corresponding function $G_{d=k}(x, u)$ that also count vertices of degree $k$ can be easily computed:

$$
G(x)=e^{C(x)} \quad \text { and by } \quad G_{d=k}(x, u)=e^{C_{d=k}(x, u)} .
$$

One just has to observe that an outerplanar graph uniquely decomposes into connected outerplanar graphs and that the number of vertices and the number of vertices of degree $k$ add up.

\section{Generating Functions for Series Parallel Graphs}

We recall that a series-parallel graph is a graph that does not contain a minor isomorphic to $K_{4}$. Further, every series-parallel graph is planar and there is a recursive description that can be also used to obtain relations for corresponding exponential generating functions.

In what follows we first describe series-parallel networks and then 2-connected and finally connected series parallel graphs. In all steps we will also take care of the vertex degrees.

3.1. Series Parallel Networks. A series-parallel network is a labelled graph with two distinguished vertices (or roots) that are called poles such that when we join the two poles in the case when they are not adjacent then the resulting graph is a 2-connected series-parallel graph. There is also a recursive description of seriesparallel (SP) networks: they are either a parallel composition of SP networks or a series decomposition of SP networks or just the smallest network consisting of the two poles and an edge joining them. 
We denote by

$$
D(x, y)=\sum_{n, m} d_{n, m} \frac{x^{n}}{n !} y^{m}
$$

the exponential generating function of all SP networks, more precisely, $d_{n, m}$ is the number of SP networks with $n+2$ vertices and $m$ edges, where the $n$ internal (different from the poles) vertices are labelled by $\{1,2, \ldots, n\}$. In the same way we define $S(x, y)$ that counts $\mathrm{SP}$ networks that have a series decomposition into at least two SP networks.
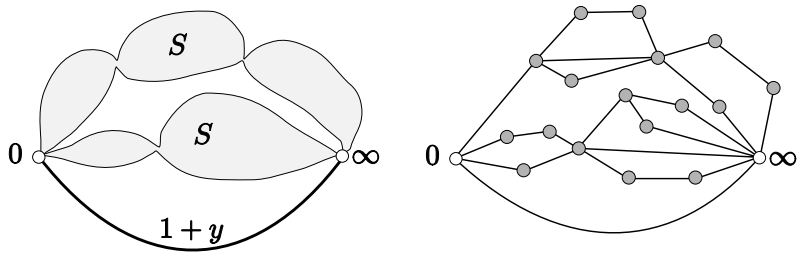

Figure 6. Parallel composition of SP networks: a scheme and an example

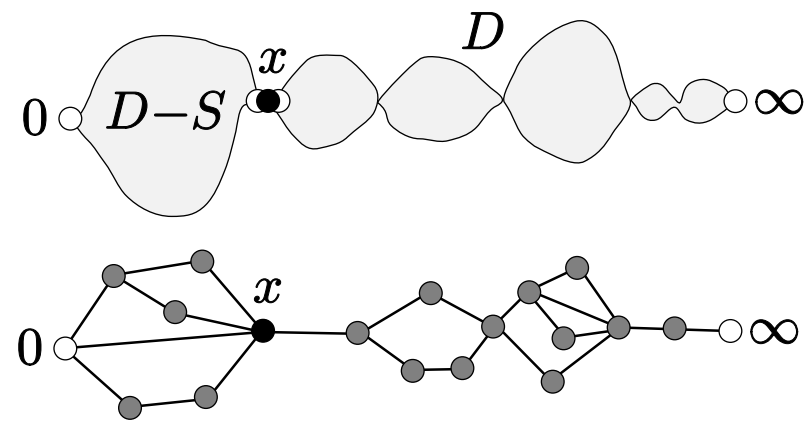

FiguRE 7. Series composition of SP networks: a scheme and an example

The recursive definition immediately translates into a system of equations for $D(x, y)$ and $S(x, y)$.

Lemma 3.1. We have

$$
\begin{aligned}
D(x, y) & =(1+y) e^{S(x, y)}-1, \\
S(x, y) & =(D(x, y)-S(x, y)) x D(x, y) .
\end{aligned}
$$

In particular, $D(x, y)$ satisfies the equation

$$
\log \left(\frac{1+D(x, y)}{1+y}\right)=\frac{x D(x, y)^{2}}{1+x D(x, y)}
$$

Proof. The fist equation (3.1) expresses the fact that a SP network is parallel composition of series networks (this is the exponential term), to which we may add or not the edge connecting the two poles. The second equation (3.2) means that a series network is formed by taking a first a non-series network (this is the term $D-S)$, and concatenating to it an arbitrary network. Since two of the poles are identified, a new internal vertex is created, hence the factor $x$. 
As in the case of outer-planar graphs we will extend these relations to generating functions where we take into account the vertex degrees. We fix some $k \geq 2$ and define by

$$
d_{i, j ; m, n ; n_{1}, n_{2}, \ldots, n_{k}, n_{\infty}}
$$

the number of SP networks with $2+n=2+n_{1}+n_{2}+\cdots+n_{k}+n_{\infty} \geq 3$ vertices and $m$ edges such that the poles have degrees $i$ and $j \in\{1,2, \ldots, k, \infty\}^{3}$ and that for $1 \leq \ell \leq k$ there are exactly $n_{\ell}$ internal vertices of degree $\ell$, and there are $n_{\infty}$ internal vertices with degree $>k$. The corresponding generating functions are then defined by

$D_{i, j}\left(x, y, z_{1}, z_{2}, \ldots, z_{k}, z_{\infty}\right)=\sum_{m, n, n_{1}, \ldots, n_{k}, n_{\infty}} d_{i, j ; m, n ; n_{1}, n_{2}, \ldots, n_{k}, n_{\infty}} y^{m} \frac{x^{n} z_{1}^{n_{1}} \cdots z_{k}^{n_{k}} z_{\infty}^{n_{\infty}}}{n !}$.

Similarly we define

$S_{i, j}\left(x, y, z_{1}, z_{2}, \ldots, z_{k}, z_{\infty}\right)=\sum_{m, n, n_{1}, \ldots, n_{k}, n_{\infty}} s_{i, j ; m, n ; n_{1}, n_{2}, \ldots, n_{k}, n_{\infty}} y^{m} \frac{x^{n} z_{1}^{n_{1}} \cdots z_{k}^{n_{k}} z_{\infty}^{n_{\infty}}}{n !}$

where we count SP networks that have a series decomposition into at least two SP networks.

The next lemma provides a system of equations for $D_{i, j}$ and $S_{i, j}$. Again, in order to state the results in a more compact form we use the convention that $\infty$ means $>k$ and $\infty-1$ means $>k-1$, in particular we set $\ell+\infty=\infty$ for all positive integers $\ell$.

Lemma 3.2. The generating functions $D_{i j}=D_{i, j}\left(x, y, z_{1}, z_{2}, \ldots, z_{k}, z_{\infty}\right)$ and $S_{i j}=S_{i, j}\left(x, y, z_{1}, z_{2}, \ldots, z_{k}, z_{\infty}\right), i, j \in\{1, \ldots, k, \infty\}$, satisfy the following system of equations:

$$
\begin{aligned}
D_{i, j} & =\sum_{r \geq 1} \sum_{i_{1}+\cdots+i_{r}=i} \sum_{j_{1}+\cdots+j_{r}=j} \frac{1}{r !} \prod_{\ell=1}^{r} S_{i_{\ell}, j_{\ell}} \\
& +y \sum_{r \geq 1} \sum_{i_{1}+\cdots+i_{r}=i-1} \sum_{j_{1}+\cdots+j_{r}=j-1} \frac{1}{r !} \prod_{\ell=1}^{r} S_{i_{\ell}, j_{\ell}} \\
S_{i, j} & =x \sum_{\ell_{1}+\ell_{2} \leq k}\left(D_{i, \ell_{1}}-S_{i, \ell_{1}}\right) z_{\ell_{1}+\ell_{2}} D_{\ell_{2}, j}+x z_{\infty} \sum_{\ell_{1}+\ell_{2}>k}\left(D_{i, \ell_{1}}-S_{i, \ell_{1}}\right) D_{\ell_{2}, j} .
\end{aligned}
$$

Proof. This is a refinement of Lemma 3.1. The first equation means that a SP network with degrees $i$ and $j$ at the poles is obtained by parallel composition of series networks whose degrees at the left and right pole sum up to $i$ and $j$, respectively; one has to distinguish according to whether the edge between the poles is added or not.

The second equation reflects the series composition. In this case only the degrees of the right pole in the first network and of the left pole in the second network have to be added.

Remark. The system provided in Lemma 3.2 is not a positive system since the equation for $S_{i, j}$ contains negative term. However, we can replace the term $D_{i, \ell_{1}}$

\footnotetext{
${ }^{3}$ Again infinite degree means degree greater than $k$.
} 
by the right hand side of (3.6). Further, note that $S_{i, \ell_{1}}$ appears in this sum so that we really end up in a positive system.

Finally, it is easy to see that this (new) system is strongly connected.

3.2. 2-Connected Series Parallel Graphs. Let $b_{n, m}$ be the number of 2connected vertex labelled SP graphs with $n$ vertices and $m$ edges and let

$$
B(x, y)=\sum_{n, m} b_{n, m} \frac{x^{n}}{n !} y^{m}
$$

be the corresponding exponential generating function. As already mentioned there is an intimate relation between SP networks and 2-connected SP graphs.

Lemma 3.3. Let $D(x, y)$ be the exponential generating function of $S P$ networks and $S(x, y)$ the exponential generating function of $S P$ networks that have a series decomposition. Then we have

$$
\frac{\partial B(x, y)}{\partial y}=\frac{x^{2}}{2} \frac{1+D(x, y)}{1+y}=\frac{x^{2}}{2} e^{S(x, y)} .
$$

Proof. A proof is given in [15]. Note that the partial derivative with respect to $y$ corresponds to rooting at an edge.

Next consider 2-connected series parallel graphs with a rooted and directed edge. In particular let $B_{i, j}=B_{i, j}\left(x, y, z_{1}, \ldots, z_{k}, z_{\infty}\right), i, j \in\{1,2, \ldots, k, \infty\}$, denote the exponential generating function of 2-connected series-parallel graphs, where the two root vertices have degrees $i$ and $j .{ }^{4}$ Note that the directed root edge connects the root vertex of degree $i$ with the other root vertex of degree $j$.

Further, let $B_{i}=B_{i}\left(x, y, z_{1}, \ldots, z_{k}, z_{\infty}\right), i \in\{2, \ldots, k, \infty\}$, be the generating function of 2-connected series parallel graphs where we just root at one vertex that has degree $i$.

Finally, let $B=B\left(x, y, z_{1}, \ldots, z_{k}, z_{\infty}\right)$ be the generating function of all 2connected SP graphs.

The next lemma quantifies the relation between SP networks and 2-connected $\mathrm{SP}$ graphs. As above we use the convention that $\infty$ means $>k$ and $\infty-1$ means $\geq k-1$, in particular we set $\ell+\infty=\infty$ for all positive integers $\ell$.

Lemma 3.4. We have

$$
\begin{aligned}
B_{i, j} & =x^{2} z_{i} z_{j} y \sum_{r \geq 1} \sum_{i_{1}+\cdots+i_{r}=i-1} \sum_{j_{1}+\cdots+j_{r}=j-1} \frac{1}{r !} \prod_{\ell=1}^{r} S_{i_{\ell}, j_{\ell}}, \\
B_{i} & =\frac{1}{i} \sum_{j=2}^{k} B_{i, j}+\frac{1}{i} B_{i, \infty} \quad(i \in\{2, \ldots, k\}) \\
B_{\infty} & =x \frac{\partial B}{\partial x}-\sum_{i=2}^{k} B_{i} \\
2 y \frac{\partial B}{\partial y} & =\sum_{i, j \in\{2, \ldots, k, \infty\}} B_{i, j}, \\
x \frac{\partial B}{\partial x} & =\sum_{i \in\{2, \ldots, k, \infty\}} B_{i} .
\end{aligned}
$$

\footnotetext{
${ }^{4}$ As above we interpret $\infty$ as $>k$.
} 
Proof. The first equation is essentially a refinement of the previous lemma. It means that 2-connected SP graphs are formed by taking parallel compositions of SP networks and adding the edge between the poles. The sum of the degrees of the poles in the networks must be one less than the degree of the resulting vertex in the SP graph.

The second equation reflects the fact that a SP graph rooted at a vertex of degree $i$ comes from a SP graph rooted at an edge whose first vertex has degree $i$, but then each of them has been counted $i$ times. The remaining equations are clear if we recall that $x \partial B / \partial x$ corresponds to graphs rooted at a vertex and $y \partial B / \partial y$ corresponds to graphs rooted at a edge (in this last case the factor 2 appears because in the definition of $B_{i, j}$ the root edge is directed).

Now let

$$
B_{d=k}(x, u)=\sum_{n, \nu} b_{n, \nu}^{(k)} \frac{x^{n}}{n !} u^{\nu}
$$

denote exponential generating function for the the numbers $b_{n, \ell}^{(k)}$ that count the number of series-parallel vertex labelled graphs with $n$ vertices, where $\nu$ vertices have degree $k$. Then we have

$$
B_{d=k}(x, u)=B(x, 1,1, \ldots, 1, u, 1) .
$$

3.3. Connected Series Parallel Graphs. The following lemma is an analogue to Lemma 2.5, whose proof also applies for series-parallel graphs.

Lemma 3.5. Let $B(x)$ be the exponential generating function of 2-connected seriesparallel graphs and $C(x)$ the corresponding exponential generating function of connected series-parallel graphs. Then we have

$$
C^{\prime}(x)=e^{B^{\prime}\left(x C^{\prime}(x)\right)},
$$

where derivatives are with respect to $x$.

Next we we introduce the generating function

$$
C_{j}\left(x, y, z_{1}, z_{2}, \ldots, z_{k}, z_{\infty}\right)=\sum_{m, n, n_{1}, \ldots, n_{k}, n_{\infty}} c_{j ; m, n ; n_{1}, \ldots, n_{k}, n_{\infty}} y^{m} \frac{x^{n} z_{1}^{n_{1}} \cdots z_{k}^{n_{k}} z_{\infty}^{n_{\infty}}}{n !},
$$

$j \in\{1,2, \ldots, k, \infty\}$, where $c_{j ; m, n, n_{1}, \ldots, n_{k}, n_{\infty}}$ is the number of labelled series parallel graphs with $n=n_{1}+\cdots+n_{k}+n_{\infty}$ vertices and $m$ edges, where one vertex of degree $j$ is marked and where $n_{\ell}$ of these $n$ vertices have degree $\ell, 1 \leq \ell \leq k$, and $n_{\infty}$ of these vertices have degree greater than $k$. Further set

$$
B_{j}^{\bullet}\left(x, y, z_{1}, z_{2}, \ldots, z_{k}, z_{\infty}\right)=\frac{1}{x z_{j}} B_{j}\left(x, y, z_{1}, z_{2}, \ldots, z_{k}, z_{\infty}\right)
$$

and

$$
C_{j}^{\bullet}\left(x, y, z_{1}, z_{2}, \ldots, z_{k}, z_{\infty}\right)=\frac{1}{x z_{j}} C_{j}\left(x, y, z_{1}, z_{2}, \ldots, z_{k}, z_{\infty}\right)
$$

Then $B_{j}^{\bullet}$ and $C_{j}^{\bullet}$ have the same interpretation as in the case of outerplanar graphs. Hence we get the same relations as stated in Lemma 2.6. The only difference is that we also take the number of edges into account, that is, we have an additional variable $y$. 
Lemma 3.6. Let $W_{j}=W_{j}\left(z_{1}, \ldots, z_{k}, z_{\infty}, C_{1}^{\bullet}, \ldots, C_{k}^{\bullet}, C_{\infty}^{\bullet}\right), j \in\{1,2, \ldots, k, \infty\}$, be defined by

$$
\begin{aligned}
W_{j} & =\sum_{i=0}^{k-j} z_{i+j} C_{j}^{\bullet}\left(x, y, z_{1}, \ldots, z_{k}, z_{\infty}\right) \\
& +z_{\infty}\left(\sum_{i=k-j+1}^{k} C_{i}^{\bullet}\left(x, y, z_{1}, \ldots, z_{k}, z_{\infty}\right)+C_{\infty}^{\bullet}\left(x, y, z_{1}, \ldots, z_{k}, z_{\infty}\right)\right), \\
W_{\infty} & =z_{\infty}\left(\sum_{i=0}^{k} C_{i}^{\bullet}\left(x, y, z_{1}, \ldots, z_{k}, z_{\infty}\right)+C_{\infty}^{\bullet}\left(x, y, z_{1}, \ldots, z_{k}, z_{\infty}\right)\right) .
\end{aligned}
$$

Then the function $C_{1}^{\bullet}, \ldots, C_{k}^{\bullet}, C_{\infty}^{\bullet}$ satisfy the system of equations

$$
\begin{aligned}
C_{j}^{\bullet}\left(x, y, z_{1}, \ldots, z_{k}, z_{\infty}\right) & =\sum_{\ell_{1}+2 \ell_{2}+3 \ell_{3}+\cdots+j \ell_{j}=j} \prod_{r=1}^{j} \frac{B_{r}^{\bullet}\left(x, y, W_{1}, \ldots, W_{k}, W_{\infty}\right)^{\ell_{r}}}{\ell_{r} !} \\
C_{\infty}^{\bullet}\left(x, z_{1}, \ldots, z_{k}, z_{\infty}\right) & =\exp \left(\sum_{j=1}^{k} B_{j}^{\bullet}\left(x, y, W_{1}, \ldots, W_{k}, W_{\infty}\right)+B_{\infty}^{\bullet}\left(x, y, W_{1}, \ldots, W_{k}, W_{\infty}\right)\right) \\
& -1-\sum_{1 \leq \ell_{1}+2 \ell_{2}+3 \ell_{3}+\cdots+k \ell_{k} \leq k} \prod_{r=1}^{k} \frac{B_{r}^{\bullet}\left(x, y, W_{1}, \ldots, W_{k}, W_{\infty}\right)^{\ell_{r}}}{\ell_{r} !} .
\end{aligned}
$$

Consequently the generating function $C_{d=k}(x, u)$ is given by

$\frac{\partial C_{d=k}(x, u)}{\partial x}=\sum_{j=1}^{k-1} C_{j}^{\bullet}(x, 1,1, \ldots, 1, u, 1)+u C_{k}^{\bullet}(x, 1,1, \ldots, 1, u, 1)+C_{\infty}^{\bullet}(x, 1,1, \ldots, 1, u, 1)$.

3.4. All Series Parallel Graphs. As in the case of outerplanar graphs the generating function $G(x)$ and the corresponding function $G_{d=k}(x, u)$ that take into account all series-parallel graphs are given by

$$
G(x)=e^{C(x)} \quad \text { and by } \quad G_{d=k}(x, u)=e^{C_{d=k}(x, u)} .
$$

\section{Generating Functions for Planar Graphs}

The number of vertices of degree 1 in planar graphs was already considered in [10], compare with Theorem 4 in [10], applied to the case where $H$ is a single edge. Therefore we will focus on vertices of degree two.

4.1. 2-Connected Planar Graphs. Our first goal is to characterize the generating function $B_{p}\left(x, y, z_{1}, z_{2}, z_{\infty}\right)$ of 2-connected planar graphs where $x$ marks vertices, $y$ edges and $z_{j}$ vertices of degree $j, j \in\{1,2, \infty\}$

First we recall a result of Walsh on 2-connected graphs without vertices of degree two. The original result [15] is stated for arbitrary labelled graphs, but it applies also to planar graphs; the reason is that a graph is planar if and only if it remains planar after removing the vertices of degree 2 . 
Lemma 4.1. Let $B_{p}(x, y)$ be the generating function for 2-connected planar graphs, and let $H_{p}(x, y)$ be the generating function for 2-connected planar graphs without vertices of degree 2 , where $x$ marks vertices and y marks edges. Also, let $D(x, y)$ be the GF of series-parallel networks as in Section 3, and B(x,y) the GF of 2connected series-parallel graphs, given by (3.8). Then

$$
H_{p}(x, D(x, y))=B_{p}(x, y)-B(x, y) .
$$

Proof. This is equation (5) from [15], here we give a sketch of the argument. Given a 2-connected planar graph, perform the following operation repeatedly: remove a vertex of degree two, if there is any, and remove parallel edges created, if any. In this way we get either a graph with minimum degree three, or a single edge in case the graph was series-parallel. This gives

$$
B_{p}(x, y)=H_{p}(x, D(x, y))+B(x, y) .
$$

Corollary4.2. The generating function $H_{p}(x, y)$ is given by

$$
H_{p}(x, y)=B_{p}(x, \phi(x, y))-B(x, \phi(x, y)),
$$

where

$$
\phi(x, y)=(1+y) \exp \left(\frac{-x y^{2}}{1+x y}\right)-1 .
$$

Proof. Since $D(x, y)$ satisfies the equation (3.3) we can express $y$ by

$$
y=\phi(x, D)=(1+D) \exp \left(\frac{-x D^{2}}{1+x D}\right)-1
$$

and obtain

$$
H_{p}(x, D)=B_{p}(x, \phi(x, D))-B(x, \phi(x, D))
$$

where we can now interpret $D$ as an independent variable.

Remark. We recall that $B_{p}(x, y)$ is determined by the system of equations (compare with [10]):

$$
\begin{aligned}
\frac{\partial B_{p}(x, y)}{\partial y} & =\frac{x^{2}}{2} \frac{1+D_{p}(x, y)}{1+y}, \\
\frac{M\left(x, D_{p}\right)}{2 x^{2} D_{p}} & =\log \left(\frac{1+D_{p}}{1+y}\right)-\frac{x D_{p}^{2}}{1+x D_{p}}, \\
M(x, y) & =x^{2} y^{2}\left(\frac{1}{1+x y}+\frac{1}{1+y}-1-\frac{(1+U)^{2}(1+V)^{2}}{(1+U+V)^{3}}\right), \\
U & =x y(1+V)^{2}, \\
V & =y(1+U)^{2} .
\end{aligned}
$$

With help of theses preliminaries we can obtain an explicit expression for $B_{p}\left(x, y, z_{1}, z_{2}, z_{\infty}\right)$.

Lemma 4.3. Let $k=2$ and let $B\left(x, y, z_{1}, z_{2}, z_{\infty}\right)$ be the generating function for 2-connected series parallel graphs and

$$
D\left(x, y, z_{1}, z_{2}, z_{\infty}\right)=\sum_{i, j \in\{1,2, \infty\}} D_{i j}\left(x, y, z_{1}, z_{2}, z_{\infty}\right)
$$


the corresponding generating function of series-parallel networks, compare with Lemma 3.2 and 3.4. Then we have

$$
B_{p}\left(x, y, z_{1}, z_{2}, z_{\infty}\right)=B\left(x, y, z_{1}, z_{2}, z_{\infty}\right)+H_{p}\left(x, D\left(x, y, z_{1}, z_{2}, z_{\infty}\right)\right) .
$$

Proof. We again use the idea of (5) from [15]. We just add the counting of vertices of degrees one and two that come from the SP networks and from SP graphs.

\subsection{Connected Planar Graphs. Set}

$$
B_{j}^{\bullet}\left(x, y, z_{1}, z_{2}, z_{\infty}\right)=\frac{1}{x} \frac{\partial B_{p}\left(x, y, z_{1}, z_{2}, z_{\infty}\right)}{\partial z_{j}} \quad(j \in\{1,2, \infty\}) .
$$

Division by $x$ is because in the definition of $B^{\bullet}$ the root bears no label.

Further, let $C_{j}^{\bullet}\left(x, y, z_{1}, z_{2}, z_{\infty}\right), j \in\{1,2, \infty\}$, denote the corresponding generating functions for connected planar graphs. Then we have (as in Lemma 2.6) the system of equations

$$
\begin{aligned}
C_{1}^{\bullet}= & B_{1}^{\bullet}\left(x, y, W_{1}, W_{2}, W_{\infty}\right), \\
C_{2}^{\bullet}= & \frac{1}{2 !}\left(B_{1}^{\bullet}\left(x, y, W_{1}, W_{2}, W_{\infty}\right)\right)^{2}+B_{2}^{\bullet}\left(x, y, W_{1}, W_{2}, W_{\infty}\right), \\
C_{\infty}^{\bullet}= & e^{B_{1}^{\bullet}\left(x, y, W_{1}, W_{2}, W_{\infty}\right)+B_{2}^{\bullet}\left(x, y, W_{1}, W_{2}, W_{\infty}\right)+B_{\infty}^{\bullet}\left(x, y, W_{1}, W_{2}, W_{\infty}\right)} \\
& -1-B_{1}^{\bullet}\left(x, y, W_{1}, W_{2}, W_{\infty}\right)-B_{2}^{\bullet}\left(x, y, W_{1}, W_{2}, W_{\infty}\right) \\
& -\frac{1}{2 !}\left(B_{1}^{\bullet}\left(x, y, W_{1}, W_{2}, W_{\infty}\right)\right)^{2},
\end{aligned}
$$

where the $W_{j}, j \in\{1,2, \infty\}$, are

$$
\begin{aligned}
W_{1} & =z_{1}+z_{2} C_{1}^{\bullet}+z_{\infty}\left(C_{2}^{\bullet}+C_{\infty}^{\bullet}\right), \\
W_{2} & =z_{2}+z_{\infty}\left(C_{1}^{\bullet}+C_{2}^{\bullet}+C_{\infty}^{\bullet}\right) \\
W_{\infty} & =z_{\infty}\left(1+C_{1}^{\bullet}+C_{2}^{\bullet}+C_{\infty}^{\bullet}\right)
\end{aligned}
$$

From this system of equations we get a single equation for

$$
C^{\bullet}=1+C_{1}^{\bullet}+C_{2}^{\bullet}+C_{\infty}^{\bullet} .
$$

if we set $z_{1}=z_{\infty}=1$, and $y=1$.

Lemma 4.4. The function $C^{\bullet}\left(x, 1,1, z_{2}, 1\right)$ satisfies a functional equation of the form

$$
C^{\bullet}=F\left(x, y, z_{2}, C^{\bullet}\right) .
$$

Proof. Since $B_{1}^{\bullet}=x z_{1}$ the equation for $C_{1}^{\bullet}$ is

$$
C_{1}^{\bullet}=x W_{1}=x\left(1+z_{2} C_{1}^{\bullet}+C_{2}^{\bullet}+C_{\infty}^{\bullet}\right)=x C^{\bullet}+x\left(z_{2}-1\right) C_{1}^{\bullet},
$$

which gives

Consequently, we have

$$
C_{1}^{\bullet}=\frac{x C^{\bullet}}{1-x\left(z_{2}-1\right)}
$$

$$
W_{1}=C^{\bullet}+\left(z_{2}-1\right) C_{1}^{\bullet}=\frac{C^{\bullet}}{1-x\left(z_{2}-1\right)} .
$$

Since $W_{2}=z_{2}-1+C^{\bullet}$ and $W_{\infty}=C^{\bullet}$ we can sum the three equations for $C_{1}^{\bullet}, C_{2}^{\bullet}, C_{\infty}^{\bullet}$ and obtain

$$
C^{\bullet}=e^{B_{1}^{\bullet}\left(x, y, W_{1}, W_{2}, W_{\infty}\right)+B_{2}^{\bullet}\left(x, y, W_{1}, W_{2}, W_{\infty}\right)+B_{\infty}^{\bullet}\left(x, y, W_{1}, W_{2}, W_{\infty}\right)}
$$


which is now a single equation for $C^{\bullet}$.

Finally the generating function $C_{d=2}(x, u)$ for connected planar graphs is determined by

$$
\frac{\partial C_{d=2}(x, u)}{\partial x}=C_{1}^{\bullet}(x, 1,1, u, 1)+u C_{2}^{\bullet}(x, 1,1, u, 1)+C_{\infty}^{\bullet}(x, 1,1, u, 1) .
$$

4.3. All Planar Graphs. As before the generating function $G(x)$ and the corresponding function $G_{d=2}(x, u)$ are given by

$$
G(x)=e^{C(x)} \quad \text { and by } \quad G_{d=2}(x, u)=e^{C_{d=k}(x, u)} .
$$

\section{Transfer of Singularities}

The main objective of this section is to consider analytic functions $f(x, u)$ that have a local representation

$$
f(x, u)=g(x, u)-h(x, u) \sqrt{1-\frac{x}{\rho(u)}},
$$

that holds in a (complex) neighbourhood $U \in \mathbb{C}^{2}$ of $\left(x_{0}, u_{0}\right)$ with $x_{0} \neq 0, u_{0} \neq 0$ and with $\rho\left(u_{0}\right)=x_{0}$ (we only have to cut off the half lines $\{x \in \mathbb{C}: \arg (x-\rho(u))=0\}$ in order to have an unambiguous value of the square root). The reason for the negative sign in front of $h(x, u)$ is that the coefficients of $\sqrt{1-x}$ are negative.

The functions $g(x, u)$ and $h(x, u)$ are analytic in $U$ and $\rho(u)$ is analytic in a neighbourhood of $u_{0}$. In our context we usually can assume that $x_{0}$ and $u_{0}$ are positive real numbers. In Section 6 we will show that solutions $f(x, u)$ of functional equations have (usually) a local expansion of this form.

Note that a function $f(x, u)$ of the form (5.1) can be also represented as

$$
f(x, u)=\sum_{\ell \geq 0} a_{\ell}(u)\left(1-\frac{x}{\rho(u)}\right)^{\ell / 2}
$$

where

$$
g(x, u)=\sum_{k \geq 0} a_{2 k}(u)\left(1-\frac{x}{\rho(u)}\right)^{k}=\sum_{k \geq 0}(-1)^{k} a_{2 k}(u) \rho(u)^{-k}(x-\rho(u))^{k}
$$

and

$$
h(x, u)=\sum_{k \geq 0} a_{2 k+1}(u)\left(1-\frac{x}{\rho(u)}\right)^{k}=\sum_{k \geq 0}(-1)^{k} a_{2 k+1}(u) \rho(u)^{-k}(x-\rho(u))^{k} .
$$

In particular, the coefficients $a_{\ell}(u)$ are analytic in $u$ (for $u$ close to $u_{0}$ ) and the power series

$$
\sum_{\ell \geq 0} a_{\ell}(u) X^{\ell}
$$

converges uniformly and absolutely if $u$ is close to $u_{0}$ and $|X|<r$ (for some properly chosen $r>0$ ). In particular, it represents an analytic function of $u$ and $X$ (in that range).

In what follows we will work with functions that have a singular expansion of the form (5.1). 
Lemma 5.1. Suppose that $f(x, u)$ has a singular expansion of the form (5.1) and that $G(x, y, z)$ is a function that is analytic at $\left(x_{0}, u_{0}, f\left(x_{0}, u_{0}\right)\right)$ such that

$$
G_{z}\left(x_{0}, y_{0}, f\left(x_{0}, u_{0}\right)\right) \neq 0 \text {. }
$$

Then

$$
\bar{f}(x, u)=G(x, u, f(x, u))
$$

has the same kind of singular expansion, that is

$$
\bar{f}(x, u)=\bar{g}(x, u)-\bar{h}(x, u) \sqrt{1-\frac{x}{\rho(u)}} .
$$

for certain analytic functions $\bar{g}(x, u)$ and $\bar{h}(x, u)$.

Proof. We use the Taylor series expansion of $G(x, u, z)$ at $z=g(x, u)$,

$$
G(x, y, z)=\sum_{\ell=0}^{\infty} G_{\ell}(x, u)(z-g(x, u))^{\ell},
$$

and substitute $z=f(x, u)$ :

$$
\begin{aligned}
\bar{f}(x, u) & =G(x, y, f(x, u)) \\
& =\sum_{\ell=0}^{\infty} G_{\ell}(x, u)\left(-h(x, u) \sqrt{1-\frac{x}{\rho(u)}}\right)^{\ell} \\
& =\sum_{k=0}^{\infty} G_{2 k}(x, u)\left(-h(x, u) \sqrt{1-\frac{x}{\rho(u)}}\right)^{2 k} \\
& +\sum_{k=0}^{\infty} G_{2 k+1}(x, u)\left(-h(x, u) \sqrt{1-\frac{x}{\rho(u)}}\right)^{2 k+1} \\
& =\bar{g}(x, u)-\bar{h}(x, u) \sqrt{1-\frac{x}{\rho(u)}} .
\end{aligned}
$$

where $\bar{g}(x, u)$ and $\bar{h}(x, u)$ are analytic at $\left(x_{0}, u_{0}\right)$. (Note that all $G_{\ell}(x, u)$ are analytic in $(x, u)$ and all appearing series are absolutely convergent.)

Lemma 5.2. Suppose that $f(x, u)$ has a singular expansion of the form (5.1) such that $|\rho(u)|$ is the radius of convergence of the function $x \mapsto f(x, u)$ if $u$ is sufficiently close to $u_{0}$. Then the partial derivative $f_{x}(x, u)$ and the integral $\int_{0}^{x} f(t, u) d t$ have local singular expansions of the form

$$
f_{x}(x, u)=\frac{g_{2}(x, u)}{\sqrt{1-\frac{x}{\rho(u)}}}+h_{2}(x, u)
$$

and

$$
\int_{0}^{x} f(t, u) d t=g_{3}(x, u)+h_{3}(x, u)\left(1-\frac{x}{\rho(u)}\right)^{3 / 2}
$$

where $g_{2}(x, u), g_{3}(x, u), h_{2}(x, u)$, and $h_{3}(x, u)$, are analytic at $\left(x_{0}, u_{0}\right)$.

Note. Now we choose the signs in front of $g_{2}(x, u)$ and $h_{3}(x, u)$ to be positive since $1 / \sqrt{1-x}$ and $(1-x)^{3 / 2}$ have positive coefficients. 
Proof. First, from (5.1) one directly derives

$$
\begin{aligned}
f_{x}(x, u) & =g_{x}(x, u)-h_{x}(x, u) \sqrt{1-\frac{x}{\rho(u)}}+\frac{h(x, u)}{2 \rho(u) \sqrt{1-\frac{x}{\rho(u)}}} \\
& =\frac{\frac{h(x, u)}{2 \rho(u)}-h_{x}(x, u)\left(1-\frac{x}{\rho(u)}\right)}{\sqrt{1-\frac{x}{\rho(u)}}}+g_{x}(x, u) \\
& =\frac{g_{2}(x, u)}{\sqrt{1-\frac{x}{\rho(u)}}}+h_{2}(x, u) .
\end{aligned}
$$

The proof of the representation of the integral is a little bit more involved. We represent $f(x, u)$ in the form

$$
f(x, u)=\sum_{j=0}^{\infty} a_{j}(u)\left(1-\frac{x}{\rho(u)}\right)^{j / 2} .
$$

Recall that the power series

$$
\sum_{j=0}^{\infty} a_{j}(u) X^{\ell}
$$

converges absolutely and uniformly in a complex neighbourhood of $u_{0}:\left|u-u_{0}\right| \leq r$ and for $|X| \leq r$ (for some $r>0$ ). Hence, there exist $\eta>0$ such that $\eta|\rho(u)|<r$ for all $u$ with $\left|u-u_{0}\right| \leq r$. Further, by assumption, there are no singularities of $f(x, u)$ in the range $|x| \leq|\rho(u)|(1-\eta)$.

We now assume that $x$ is close to $x_{0}$ so that $|1-x / \rho(u)|<r$. Then we split up the integral $\int_{0}^{x} f(t, u) d t$ into three parts:

$$
\begin{aligned}
\int_{0}^{x} f(t, u) d t & =\int_{0}^{\rho(u)(1-\eta)} f(t, u) d t+\int_{\rho(u)(1-\eta)}^{\rho(u)} f(t, u) d t+\int_{\rho(u)}^{x} f(t, u) d t \\
& =I_{1}(u)+I_{2}(u)+I_{3}(x, u) .
\end{aligned}
$$

Since we have chosen $\eta$ is a proper way there are no singularities of $f(t, u)$ in the range $|t| \leq|\rho(u)|(1-\eta)$. Hence, $I_{1}(u)$ is an analytic function in $u$.

Next, by using the series representation (5.5) we obtain

$$
\begin{aligned}
I_{2}(u) & =\int_{\rho(u)(1-\eta)}^{\rho(u)} \sum_{j=0}^{\infty} a_{j}(u)\left(1-\frac{t}{\rho(u)}\right)^{j / 2} d t \\
& =-\left.\sum_{j=0}^{\infty} a_{j}(u) \frac{2 \rho(u)}{j+1}\left(1-\frac{t}{\rho(u)}\right)^{\frac{j+2}{2}}\right|_{t=\rho(u)(1-\eta)} ^{t=\rho(u)} \\
& =\sum_{j=0}^{\infty} a_{j}(u) \frac{2 \rho(u)}{j+1} \eta^{\frac{j+2}{2}},
\end{aligned}
$$

that represents an analytic function, too. 
Finally, the third integral evaluates to

$$
\begin{aligned}
I_{3}(x, u) & =\int_{\rho(u)}^{x} \sum_{j=0}^{\infty} a_{j}(u)\left(1-\frac{t}{\rho(u)}\right)^{j / 2} d t \\
& =-\left.\sum_{j=0}^{\infty} a_{j}(u) \frac{2 \rho(u)}{j+1}\left(1-\frac{t}{\rho(u)}\right)^{\frac{j+2}{2}}\right|_{t=\rho(u)} ^{t=x} \\
& =-\sum_{j=0}^{\infty} a_{j}(u) \frac{2 \rho(u)}{j+1}\left(1-\frac{x}{\rho(u)}\right)^{\frac{j+2}{2}} .
\end{aligned}
$$

Of course, this can be represented as

$$
I_{3}(x, u)=\tilde{g}(x, u)+\tilde{h}(x, u)\left(1-\frac{x}{\rho(u)}\right)^{3 / 2}
$$

with analytic function $\tilde{g}(x, u)$ and $\tilde{h}(x, u)$. Putting these three representations together we directly get (5.4).

Another important feature is that we can switch between local expansions in terms of $x$ and $u$.

Lemma 5.3. Suppose that $f(x, u)$ has a local representation of the form (5.1) such that

$$
\rho\left(u_{0}\right) \neq 0 \quad \text { and } \quad \rho^{\prime}\left(u_{0}\right) \neq 0 .
$$

Then the singular expansion (5.1) can be rewritten as

$$
f(x, u)=\tilde{g}(x, u)-\tilde{h}(x, u) \sqrt{1-\frac{u}{R(x)}},
$$

where $R(x)$ is the (analytic) inverse function of $\rho(u)$.

Proof. Since $\rho^{\prime}\left(u_{0}\right) \neq 0$ it follows from the Weierstrass preparation theorem ${ }^{5}$ that there exists an analytic function $K(x, u)$ with $K\left(x_{0}, u_{0}\right) \neq 0$ such that

$$
\rho(u)-x=K(x, u)(R(x)-u)
$$

where $R(x)$ is the (analytic) inverse function of $\rho(u)$ in a neighbourhood of $x_{0}$. This is because near $\left(x_{0}, u_{0}\right)$ we have $R(x)=u$ if and only if $\rho(u)=x$.

Consequently

$$
1-\frac{x}{\rho(u)}=K(x, u) \frac{R(x)}{\rho(u)}\left(1-\frac{u}{R(x)}\right)
$$

\footnotetext{
${ }^{5}$ The Weierstrass preparation theorem (see [13] or [9, Theorem B.5]) says that every non-zero function $F\left(z_{1}, \ldots, z_{d}\right)$ with $F(0, \ldots, 0)=0$ that is analytic at $(0, \ldots, 0)$ has a unique factorization $F\left(z_{1}, \ldots, z_{d}\right)=K\left(z_{1}, \ldots, z_{d}\right) W\left(z_{1} ; z_{2}, \ldots, z_{d}\right)$ into analytic factors, where $K(0, \ldots, 0) \neq 0$ and $W\left(z_{1} ; z_{2}, \ldots, z_{d}\right)=z_{1}^{d}+z_{1}^{d-1} g_{1}\left(z_{2}, \ldots, z_{d}\right)+\cdots+g_{d}\left(z_{2}, \ldots, z_{d}\right)$ is a so-called Weierstrass polynomial, that is, all $g_{j}$ are analytic and satisfy $g_{j}(0, \ldots, 0)=0$.

We use here a shifted version and apply it for $d=1$ which constitutes a refined version of the implicit function theorem.
} 
and also

$$
\begin{aligned}
f(x, u) & =g(x, u)-h(x, u) \sqrt{K(x, u) \frac{R(x)}{\rho(u)}} \sqrt{1-\frac{u}{R(x)}} \\
& =\tilde{g}(x, u)-\tilde{h}(x, u) \sqrt{1-\frac{u}{R(x)}}
\end{aligned}
$$

Remark. Note that Lemma 5.3 has some flexibility. For example, if $f(x, u)$ has a singular expansion of the form

$$
f(x, u)=g(x, u)+h(x, u)\left(1-\frac{x}{\rho(u)}\right)^{3 / 2}
$$

then we also get a singular expansion of the form

$$
f(x, u)=\tilde{g}(x, u)+\tilde{h}(x, u)\left(1-\frac{u}{R(x)}\right)^{\frac{3}{2}}
$$

Similarly, if $f(x, u)$ is of the form

$$
f(x, u)=\frac{g_{2}(x, u)}{\sqrt{1-\frac{x}{\rho(u)}}}+h_{2}(x, u)
$$

then we can rewrite this to

$$
f(x, u)=\frac{\tilde{g}_{2}(x, u)}{\sqrt{1-\frac{u}{R(x)}}}+\tilde{h}_{2}(x, u) .
$$

If we combine Lemma 5.2 and Lemma 5.3 we thus get the following result, which is fundamental for the proofs of our main results later on.

Theorem 5.4. Suppose that $f(x, u)$ has a singular expansion of the form (5.1) such that $|\rho(u)|$ is the radius of convergence of the function $x \mapsto f(x, u)$ if $u$ is sufficiently close to $u_{0}$, and $\rho(u)$ satisfies $\rho\left(u_{0}\right) \neq 0$ and $\rho^{\prime}\left(u_{0}\right) \neq 0$. Then the partial derivative $f_{u}(x, u)$ and the integral $\int_{0}^{u} f(x, t) d t$ have local singular expansions of the form

$$
f_{u}(x, u)=\frac{g_{2}(x, u)}{\sqrt{1-\frac{x}{\rho(u)}}}+h_{2}(x, u)
$$

and

$$
\int_{0}^{u} f(x, t) d t=g_{3}(x, u)+h_{3}(x, u)\left(1-\frac{x}{\rho(u)}\right)^{\frac{3}{2}},
$$

where $g_{2}(x, u), g_{3}(x, u), h_{2}(x, u)$, and $h_{3}(x, u)$, are analytic at $\left(x_{0}, u_{0}\right)$.

Proof. For the proof of both (5.6) and (5.7), we apply first Lemma 5.3 and switch to a singular expansion in terms of $\sqrt{1-u / R(x)}$. Then we apply Lemma 5.2 in order to get an expansion for the derivative or the integral, and finally we apply Lemma 5.3 again in order to get back to an expansion in terms of $\sqrt{1-x / \rho(u)}$. 


\section{Systems of Functional Equations}

The purpose of this section is provide a tool box for proving central limit theorems with the help of generating functions that satisfy a system of equations. Most results of this section are based on the methods of [5] (compare also with [4]). However, we provide a more structured presentation. Furthermore we extend the previous results by proper tail estimates.

Let us start with a simple observation. Suppose that $y=y(x)$ is an analytic generating function that satisfies the functional equation

$$
y=F(x, y),
$$

where $F(x, y)$ is an analytic function in $x$ and $y$ around $x=y=0$. Further suppose that there exist $x=x_{0}$ and $y=y_{0}=y\left(x_{0}\right)$ that are solutions of the system of equations

$$
\begin{aligned}
& y=F(x, y), \\
& 1=F_{y}(x, y),
\end{aligned}
$$

where $F(x, y)$ is analytic and we have $F_{x}\left(x_{0}, y_{0}\right) \neq 0$ and $F_{y y}\left(x_{0}, y_{0}\right) \neq 0$.

If we consider the equation $y-F(x, y)=0$ around $x=x_{0}$ and $y=y_{0}$, then we have $1-F_{y}\left(x_{0}, y_{0}\right)=0$ and $-F_{y y}\left(x_{0}, y_{0}\right) \neq 0$. Hence by the Weierstrass preparation theorem (see $[9,13])$ there exist functions $K(x, y), p(x), q(x)$ which are analytic around $x=x_{0}$ and $y=y_{0}$ and satisfy $K\left(x_{0}, y_{0}\right) \neq 0, p\left(x_{0}\right)=q\left(x_{0}\right)=0$ and

$$
y-F(x, y)=K(x, y)\left(\left(y-y_{0}\right)^{2}+p(x)\left(y-y_{0}\right)+q(x)\right)
$$

locally around $x=x_{0}$ and $y=y_{0}$. Since $F_{x}\left(x_{0}, y_{0}\right) \neq 0$ we also have $q_{x}\left(x_{0}\right) \neq 0$. This means that any analytic function $y=y(x)$ which satisfies $y(x)=F(x, y(x))$ in a subset of a neighbourhood of $x=x_{0}$ with $x_{0}$ on its boundary and is given by

$$
y(x)=y_{0}-\frac{p(x)}{2} \pm \sqrt{\frac{p(x)^{2}}{4}-q(x)} .
$$

Since $p\left(x_{0}\right)=0$ and $q_{x}\left(x_{0}\right) \neq 0$ we have

$$
\frac{\partial}{\partial x}\left(\frac{p(x)^{2}}{4}-q(x)\right)_{x=x_{0}} \neq 0,
$$

too. Thus there exists an analytic function $K_{2}(x)$ such that $K_{2}\left(x_{0}\right) \neq 0$ and

$$
\frac{p(x)^{2}}{4}-q(x)=K_{2}(x)\left(x-x_{0}\right)
$$

locally around $x=x_{0}$. This finally leads to a local representation of $y=y(x)$ of the kind

$$
y(x)=g(x)-h(x) \sqrt{1-\frac{x}{x_{0}}},
$$

in which $g(x)$ and $h(x)$ are analytic around $x=x_{0}$ and satisfy $g\left(x_{0}\right)=y_{0}$ and $h\left(x_{0}\right) \neq 0$.

It is easy to extend the above considerations, where we have an additional (analytic) parameter $u$ and we are searching for a solution $y=y(x, u)$ of an equation of the form $y=F(x, y, u)$. Then we get an representation of the form

$$
y(x, u)=g(x, u)-h(x, u) \sqrt{1-\frac{x}{x_{0}(u)}}
$$

which is exactly of the form (5.1). 
Next we state a generalization of the one-dimensional case, where we assume additionally that the parameter $\mathbf{u}$ is multivariate and where we also assume that the coefficients of the generating functions that appear are non-negative. Let $\mathbf{F}(x, \mathbf{y}, \mathbf{u})=\left(F_{1}(x, \mathbf{y}, \mathbf{u}), \ldots, F_{N}(x, \mathbf{y}, \mathbf{u})\right)^{\mathrm{T}}$ be a column vector ${ }^{6}$ of functions $F_{j}(x, \mathbf{y}, \mathbf{u}), 1 \leq j \leq N$, with complex variables $x, \mathbf{y}=\left(y_{1}, \ldots, y_{N}\right)^{\mathrm{T}}, \mathbf{u}=$ $\left(u_{1}, \ldots, u_{k}\right)^{\mathrm{T}}$ which are analytic around 0 and satisfy $F_{j}(0, \mathbf{0}, \mathbf{0})=0$ for $1 \leq j \leq N$. We are interested in the analytic solution $\mathbf{y}=\mathbf{y}(x, \mathbf{u})=\left(y_{1}(x, \mathbf{u}), \ldots, y_{N}(x, \mathbf{u})\right)^{\mathrm{T}}$ of the functional equation

$$
\mathbf{y}=\mathbf{F}(x, \mathbf{y}, \mathbf{u})
$$

with $\mathbf{y}(0, \mathbf{0})=\mathbf{0}$, i.e., we demand that the (unknown) functions $y_{j}=y_{j}(x, \mathbf{u})$, $1 \leq j \leq N$, satisfy the system of functional equations

$$
\begin{aligned}
y_{1} & =F_{1}\left(x, y_{1}, y_{2}, \ldots, y_{N}, \mathbf{u}\right), \\
y_{2} & =F_{2}\left(x, y_{1}, y_{2}, \ldots, y_{N}, \mathbf{u}\right), \\
& \vdots \\
y_{N} & =F_{N}\left(x, y_{1}, y_{2}, \ldots, y_{N}, \mathbf{u}\right) .
\end{aligned}
$$

It is convenient to define the notion of a dependency (di)graph $G_{\mathbf{F}}=(V, E)$ for such a system of functional equations $\mathbf{y}=\mathbf{F}(x, \mathbf{y}, \mathbf{u})$. The vertices $V=$ $\left\{y_{1}, y_{2}, \ldots, y_{N}\right\}$ are just the unknown functions and an ordered pair $\left(y_{i}, y_{j}\right)$ is contained in the edge set $E$ if and only if $F_{i}(x, \mathbf{y}, \mathbf{u})$ really depends on $y_{j}$.

If the functions $F_{j}(x, \mathbf{y}, \mathbf{u})$ have non-negative Taylor coefficients then it is easy to see that the solutions $y_{j}(x, \mathbf{u})$ have the same property. (One only has to solve the system iteratively by setting $\mathbf{y}_{0}(x, \mathbf{u})=0$ and $\mathbf{y}_{i+1}(x, \mathbf{u})=\mathbf{F}\left(x, \mathbf{y}_{i}(x, \mathbf{u})\right.$, $\left.\mathbf{u}\right)$ for $i \geq 0$. The limit $\mathbf{y}(x, \mathbf{u})=\lim _{i \rightarrow \infty} \mathbf{y}_{i}(x, \mathbf{u})$ is the (unique) solution of the system above.)

Proposition 6.1. Let $\mathbf{F}(x, \mathbf{y}, \mathbf{u})=\left(F_{1}(x, \mathbf{y}, \mathbf{u}), \ldots, F_{N}(x, \mathbf{y}, \mathbf{u})\right)^{\mathrm{T}}$ be functions analytic around $x=0, \quad \mathbf{y}=\left(y_{1}, \ldots, y_{N}\right)^{\mathrm{T}}=\mathbf{0}, \quad \mathbf{u}=\left(u_{1}, \ldots, u_{k}\right)^{\mathrm{T}}=\mathbf{0}$, whose Taylor coefficients are all non-negative, such that $\mathbf{F}(0, \mathbf{y}, \mathbf{u})=\mathbf{0}, \mathbf{F}(x, \mathbf{0}, \mathbf{u}) \neq \mathbf{0}$, $\mathbf{F}_{x}(x, \mathbf{y}, \mathbf{u}) \neq \mathbf{0}$, and such that there exists $j$ with $\mathbf{F}_{y_{j} y_{j}}(x, \mathbf{y}, \mathbf{u}) \neq \mathbf{0}$. Furthermore assume that the dependency graph of $\mathbf{F}$ is strongly connected and that the region of convergence of $\mathbf{F}$ is large enough that there exists a complex neighbourhood $U$ of $\mathbf{u}=\mathbf{1}=(1, \ldots, 1)$ where the system

$$
\begin{aligned}
& \mathbf{y}=\mathbf{F}(x, \mathbf{y}, \mathbf{u}), \\
& 0=\operatorname{det}\left(\mathbf{I}-\mathbf{F}_{\mathbf{y}}(x, \mathbf{y}, \mathbf{u})\right) .
\end{aligned}
$$

has solutions $x=x_{0}(\mathbf{u})$ and $\mathbf{y}=\mathbf{y}_{0}(\mathbf{u})$ that are positive and real for real $\mathbf{u}$.

Let

$$
\mathbf{y}=\mathbf{y}(x, \mathbf{u})=\left(y_{1}(x, \mathbf{u}), \ldots, y_{N}(x, \mathbf{u})\right)^{\mathrm{T}}
$$

denote the analytic solutions of the system

$$
\mathbf{y}=\mathbf{F}(x, \mathbf{y}, \mathbf{u})
$$

with $\mathbf{y}(0, \mathbf{u})=\mathbf{0}$ and assume that $d_{n, j}>0(1 \leq j \leq N)$ for $n \geq n_{1}$, where $y_{j}(x, \mathbf{1})=\sum_{n \geq 0} d_{n, j} x^{n}$.

\footnotetext{
${ }^{6}$ We denote the transpose of a vector $v$ by $v^{\mathrm{T}}$.
} 
Then there exits $\varepsilon>0$ such that $y_{j}(x, \mathbf{u})$ admit a representation of the form

$$
y_{j}(x, \mathbf{u})=g_{j}(x, \mathbf{u})-h_{j}(x, \mathbf{u}) \sqrt{1-\frac{x}{x_{0}(\mathbf{u})}}
$$

for $\mathbf{u} \in U$ and $\left|x-x_{0}(\mathbf{u})\right|<\varepsilon$, where $g_{j}(x, \mathbf{u}) \neq 0$ and $h_{j}(x, \mathbf{u}) \neq 0$ are analytic functions with $\left(g_{j}\left(x_{0}(\mathbf{u}), \mathbf{u}\right)\right)_{j}=\left(y_{j}\left(x_{0}(\mathbf{u}), \mathbf{u}\right)\right)_{j}=\mathbf{y}_{0}(\mathbf{u})$. Furthermore, there exists $\delta>0$ such that $y_{j}(x, \mathbf{u})$ is analytic in $(x, \mathbf{u})$ for $\mathbf{u} \in U$ and $\left|x-x_{0}(\mathbf{u})\right| \geq \varepsilon$ but $|x| \leq\left|x_{0}(\mathbf{u})\right|+\delta$ (this condition guarantees that $\mathbf{y}(x, \mathbf{u})$ has a unique smallest singularity with $\left.|x|=\left|x_{0}(\mathbf{u})\right|\right)$.

Proof. A proof is given in [5].

With the help of Lemma 5.1 we immediately derive the following

Corollary6.2. Suppose that $G(x, \mathbf{y}, \mathbf{u})$ is a power series such that $\left(x_{0}(\mathbf{1}), \mathbf{y}_{0}(\mathbf{1}), \mathbf{1}\right)$ is an inner point of the region of convergence of $G(x, \mathbf{y}, \mathbf{u})$ and that $G_{\mathbf{y}}\left(x_{0}(\mathbf{1}), \mathbf{y}_{0}(\mathbf{1}), \mathbf{1}\right) \neq \mathbf{0}$.

Then $G(x, \mathbf{y}(x, \mathbf{u}), \mathbf{u})$ has a representation of the form

$$
G(x, \mathbf{y}(x, \mathbf{u}), \mathbf{u})=g(x, \mathbf{u})-h(x, \mathbf{u}) \sqrt{1-\frac{x}{x_{0}(\mathbf{u})}}
$$

for $\mathbf{u} \in U$ and $\left|x-x_{0}(\mathbf{u})\right|<\varepsilon$, where $g(x, \mathbf{u}) \neq 0$ and $h(x, \mathbf{u}) \neq 0$ are analytic functions. Furthermore, $G(x, \mathbf{y}(x, \mathbf{u}), \mathbf{u})$ is analytic in $(x, \mathbf{u})$ for $\mathbf{u} \in U$ and $\mid x-$ $x_{0}(\mathbf{u}) \mid \geq \varepsilon$ but $|x| \leq\left|x_{0}(\mathbf{u})\right|+\delta$.

An essential assumption of Proposition 6.1 is that $\left(x_{0}(\mathbf{1}), \mathbf{y}_{0}(\mathbf{1}), \mathbf{1}\right)$ is a regular point of $\mathbf{F}(x, \mathbf{y}, \mathbf{u})$. However, this is not always satisfied. The following Proposition discusses a situation where one has a square-root singularity of a specific kind at the critical point.

Proposition 6.3. Suppose that $F(x, y, u)$ has a local representation of the form

$$
F(x, y, u)=g(x, y, u)+h(x, y, u)\left(1-\frac{y}{r(x, u)}\right)^{3 / 2}
$$

with functions $g(x, y, u), h(x, y, u), r(x, u)$ that are analytic around $\left(x_{0}, y_{0}, u_{0}\right)$ and satisfy $g_{y}\left(x_{0}, y_{0}, u_{0}\right) \neq 1, h\left(x_{0}, y_{0}, u_{0}\right) \neq 0, r\left(x_{0}, u_{0}\right) \neq 0$, and $r_{x}\left(x_{0}, u_{0}\right) \neq$ $g_{x}\left(x_{0}, y_{0}, u_{0}\right)$. Furthermore, suppose that $y=y(x, u)$ is a solution of the functional equation

$$
y=F(x, y, u)
$$

with $y\left(x_{0}, u_{0}\right)=y_{0}$. Then $y(x, u)$ has a local representation of the form

$$
y(x, u)=g_{1}(x, u)+h_{1}(x, u)\left(1-\frac{x}{\rho(u)}\right)^{3 / 2}
$$

where $g_{1}(x, u), h_{1}(x, u)$ and $\rho(u)$ are analytic at $\left(x_{0}, u_{0}\right)$ and satisfy $h_{1}\left(x_{0}, u_{0}\right) \neq 0$ and $\rho\left(u_{0}\right)=x_{0}$.

Proof. Set $Y=(1-y / r(x, u))^{1 / 2}$. Then $F(x, y, u)$ can be represented as

$$
F(x, y, u)=A_{0}(x, u)+A_{2}(x, u) Y^{2}+A_{3}(x, u) Y^{3}+A_{4}(x, u) Y^{4}+\cdots,
$$


where $A_{k}(x, u)$ are analytic functions; compare with (5.2). If we now consider the equation $y=F(x, y, u)$ and replace the left hand side by $y=r(x, u)\left(1-Y^{2}\right)$, we get

$$
r(x, u)-A_{0}(x, u)=\left(r(x, u)+A_{2}(x, u)\right) Y^{2}+A_{3}(x, u) Y^{3}+A_{4}(x, u) Y^{4}+\cdots .
$$

Since $r\left(x_{0}, u_{0}\right)=A_{0}\left(x_{0}, u_{0}\right)=g\left(x_{0}, y_{0}, u_{0}\right)$ and $r_{x}\left(x_{0}, u_{0}\right) \neq A_{0, x}\left(x_{0}, u_{0}\right)=$ $g_{x}\left(x_{0}, y_{0}, u_{0}\right)$, again by the preparation theorem there exist analytic functions $K(x, u)$ and $\rho(u)$, with $K\left(x_{0}, u_{0}\right) \neq 0$ and $\rho\left(u_{0}\right)=x_{0}$, such that locally around $\left(x_{0}, u_{0}\right)$

$$
r(x, u)-A_{0}(x, u)=K(x, u)(x-\rho(u)) .
$$

Hence if we set $X=(1-x / \rho(u))^{1 / 2}$ and $L(x, u)=(-K(x, u) \rho(u))^{1 / 2}$ we get

$$
L(x, u)^{2} X^{2}=Y^{2}\left(r(x, u)+A_{2}(x, u)+A_{3}(x, u) Y+A_{4}(x, u) Y^{2} \cdots\right) .
$$

or

$$
L(x, u) X=B_{1}(x, u) Y+B_{2}(x, u) Y^{2}+B_{3}(x, u) Y^{3}+\cdots,
$$

where $B_{1}(x, u)=\left(r(x, u)+A_{2}(x, u)\right)^{1 / 2}$ and $B_{\ell}(x, u)$ are suitably chosen analytic functions. Furthermore, since $L(x, u) \neq 0$ and $B_{1}(x, u) \neq 0$ in a neighbourhood of $\left(x_{0}, u_{0}\right)$, we can invert this relation and get

$$
Y=\frac{L(x, u)}{B_{1}(x, u)} X+C_{2}(x, u) X^{2}+C_{3}(x, u) X^{3}+\cdots .
$$

By squaring this equation and substituting $Y^{2}=1-y / r(x, u)$, we finally obtain the representation

$$
1-\frac{y}{r(x, u)}=\frac{L(x, u)^{2}}{B_{1}(x, u)^{2}} X^{2}+D_{3}(x, u) X^{3}+D_{4}(x, u) X^{4}+\cdots
$$

which can be rewritten into the form (6.11). Since $h_{1}\left(x_{0}, u_{0}\right)=r\left(x_{o}, u_{0}\right) D_{3}\left(x_{0}, u_{0}\right)$, we only need to check that $D_{3}\left(x_{0}, u_{0}\right) \neq 0$. But $D_{3}=2 B_{2} L^{2} / B_{1}^{2}$ and $B_{2}=$ $A_{3} /\left(2 \sqrt{r+A_{2}}\right)$, and now it is enough to recall that $L\left(x_{0}, u_{0}\right) \neq 0$ and $A_{3}\left(x_{0}, u_{0}\right) \neq$ 0 .

Now suppose that $\mathbf{y}(x, \mathbf{u})$ is a solution of a system of equation as in Proposition 6.1. Further let $G(x, \mathbf{y}, \mathbf{u})$ be a power series with non-negative Taylor coefficients at $(0, \mathbf{0}, \mathbf{0})$ such that $\left(x_{0}(\mathbf{1}), \mathbf{y}_{0}(\mathbf{1}), \mathbf{1}\right)$ is an inner point of the region of convergence of $G(x, \mathbf{y}, \mathbf{u})$. Then

$$
G(x, \mathbf{y}(x, \mathbf{u}), \mathbf{u})=\sum_{n, \mathbf{m}} c_{n, \mathbf{m}} x^{n} \mathbf{u}^{\mathbf{m}}
$$

has non-negative coefficients $c_{n, \mathbf{m}}$, too. In fact, we also have that for every $n \geq n_{0}$ there exists $\mathbf{m}$ such that $c_{n, \mathbf{m}}>0$. In particular it follows that

$$
c_{n}(\mathbf{u})=\sum_{\mathbf{m}} c_{n, \mathbf{m}} \mathbf{u}^{\mathbf{m}}
$$

is non-zero for $n \geq n_{0}$.

Now let $\mathbf{X}_{n}=\left(X_{n}^{(1)}, \ldots, X_{n}^{(N)}\right),\left(n \geq n_{0}\right)$ denote an $N$-dimensional discrete random vector with

$$
\operatorname{Pr}\left\{\mathbf{X}_{n}=\mathbf{m}\right\}:=\frac{c_{n, \mathbf{m}}}{c_{n}} .
$$


Then the expectation $\mathbf{E} \mathbf{u}^{\mathbf{X}_{n}}=\mathbf{E} u_{1}^{X_{n}^{(1)}} \cdots u_{N}^{X_{n}^{(N)}}$ is given by

$$
\mathbf{E} \mathbf{u}^{\mathbf{X}_{n}}=\frac{c_{n}(\mathbf{u})}{c_{n}(\mathbf{1})}
$$

With the help of singularity analysis we derive an asymptotic representation for $\mathbf{E} \mathbf{u}^{\mathbf{X}_{n}}$.

Proposition 6.4. Suppose that $\mathbf{X}_{n}\left(n \geq n_{0}\right)$ is defined as above. Then we have uniformly for $\mathbf{u} \in U$

$$
\mathbf{E} \mathbf{u}^{\mathbf{X}_{n}}=\frac{h\left(x_{0}(\mathbf{u}), \mathbf{y}_{0}(\mathbf{u})\right)}{h\left(x_{0}(\mathbf{1}), \mathbf{y}_{0}(\mathbf{1})\right.}\left(\frac{x_{0}(\mathbf{1})}{x_{0}(\mathbf{u})}\right)^{n}\left(1+O\left(\frac{1}{n}\right)\right) .
$$

Proof. By applying the transfer lemma in [8], we get from (6.9) that

$$
c_{n}(\mathbf{u})=\left[x^{n}\right] G(x, \mathbf{y}(x, \mathbf{u}), \mathbf{u})=\frac{h\left(x_{0}(\mathbf{u}), \mathbf{y}_{0}(\mathbf{u})\right)}{2 \sqrt{\pi}} n^{-3 / 2} x_{0}(\mathbf{u})^{-n}\left(1+O\left(\frac{1}{n}\right)\right)
$$

uniformly for $\mathbf{u}$ in a complex neighbourhood of $\mathbf{1}$. This proves (6.13).

Next we state (and prove) a multivariate version of the so-called Quasi Power Theorem by H.-K. Hwang [11] (see also [9], similar theorems can be found in [1, 2]). Note, too, that there exists a precise large deviation result for the univariate case $[12]$.

Proposition 6.5. Let $\mathbf{X}_{n}$ be a $N$-dimensional random vector with the property that

$$
\mathbf{E} \mathbf{u}^{\mathbf{X}_{n}}=e^{\lambda_{n} \cdot A(\mathbf{u})+B(\mathbf{u})}\left(1+O\left(\frac{1}{\phi_{n}}\right)\right),
$$

holds uniformly in a complex neighborhood of $\mathbf{u}=\mathbf{1}$, where $\lambda_{n}$ and $\phi_{n}$ are sequences of positive real numbers with $\lambda_{n} \rightarrow \infty$ and $\phi_{n} \rightarrow \infty$, and $A(\mathbf{u})$ and $B(\mathbf{u})$ are analytic functions in this neighborhood of $\mathbf{u}=\mathbf{1}$ with $A(\mathbf{1})=B(\mathbf{1})=\mathbf{0}$.

Then $\mathbf{E} \mathbf{X}_{n}=\lambda_{n} \boldsymbol{\mu}+O\left(1+\lambda_{n} / \phi_{n}\right)$ and $\operatorname{Var} X_{n}=\lambda_{n} \boldsymbol{\Sigma}+O\left(1+\lambda_{n} / \phi_{n}\right)$, where $\boldsymbol{\mu}=A_{\mathbf{u}}(\mathbf{1})=\left(A_{u_{j}}(\mathbf{1})\right)_{1 \leq j \leq N}$ and $\boldsymbol{\Sigma}=\left(A_{u_{i} u_{j}}(\mathbf{1})+\delta_{i j} A_{u_{j}}(\mathbf{1})\right)_{1 \leq i, j \leq N}$. Then $\mathbf{X}_{n}$ satisfies a central limit theorem of the form

$$
\frac{1}{\sqrt{\lambda_{n}}}\left(\mathbf{X}_{n}-\mathbf{E} \mathbf{X}_{n}\right) \rightarrow N(\mathbf{0}, \boldsymbol{\Sigma}) \text {. }
$$

Finally if we additionally assume that $\lambda_{n}=\phi_{n}$, then there exist positive constants $c_{1}, c_{2}, c_{3}$ such that

$$
\operatorname{Pr}\left\{\left\|\mathbf{X}_{n}-\mathbf{E} \mathbf{X}_{n}\right\| \geq \varepsilon \sqrt{\lambda_{n}}\right\} \leq c_{1} e^{-c_{2} \varepsilon^{2}}
$$

uniformly for $\varepsilon \leq c_{3} \sqrt{\lambda_{n}}$.

Proof. For the reader's convenience we first recall a proof for the univariate case $N=1$, that is, we have

$$
\mathbf{E} u^{X_{n}}=e^{\lambda_{n} \cdot a(u)+b(u)}\left(1+O\left(\frac{1}{\phi_{n}}\right)\right) .
$$

By assumption, we obtain for $t$ in a neighborhood of $t=0$

$$
\mathbf{E} e^{i t X_{n}}=e^{i t \lambda_{n} \mu-\frac{1}{2} t^{2} \lambda_{n} \sigma^{2}+O\left(\lambda_{n} t^{3}\right)+O(t)}\left(1+O\left(\frac{1}{\phi_{n}}\right)\right) \text {. }
$$


Set $Y_{n}=\left(X_{n}-\lambda_{n} \mu\right) / \sqrt{\lambda_{n}}$, where $\mu=a^{\prime}(1)$. Then, replacing $t$ by $t / \sqrt{\lambda_{n}}$, one gets directly

$$
\mathbf{E} e^{i t Y_{n}}=e^{-\frac{\sigma^{2}}{2} t^{2}+O\left(t^{3} / \sqrt{\lambda_{n}}\right)+O\left(t / \sqrt{\lambda_{n}}\right)}\left(1+O\left(\frac{1}{\phi_{n}}\right)\right),
$$

where $\sigma^{2}=a^{\prime}(1)+a^{\prime \prime}(1)$. Thus, $Y_{n}$ is asymptotically normal with zero mean and variance $\sigma^{2}$.

Next set $f_{n}(u)=\mathbf{E} u^{X_{n}}$. Then $f_{n}^{\prime}(1)=\mathbf{E} X_{n}$. One the other hand, by Cauchy's formula, we have

$$
f_{n}^{\prime}(1)=\frac{1}{2 \pi i} \int_{|u-1|=\rho} \frac{f_{n}(u)}{(u-1)^{2}} d u .
$$

In particular, we use the circle $|u-1|=1 / \lambda_{n}$ as the path of integration and get

$$
\begin{aligned}
& \text { E } X_{n}= \\
& \frac{1}{2 \pi i} \int_{|u-1|=1 / \lambda_{n}} \frac{1+\left(\lambda_{n} a^{\prime}(1)+b^{\prime}(1)\right)(u-1)+O\left(\lambda_{n}(u-1)^{2}\right)}{(u-1)^{2}}\left(1+O\left(\frac{1}{\phi_{n}}\right)\right) d u \\
& =\lambda_{n} a^{\prime}(1)+O\left(1+\frac{\lambda_{n}}{\phi_{n}}\right) \text {. }
\end{aligned}
$$

We can treat the variance Similarly. Set $g_{n}(u)=f_{n}(u) u^{-\lambda_{n} a^{\prime}(1)-b^{\prime}(1)}$. Then $\operatorname{Var} X_{n}=g^{\prime}(1)+g^{\prime \prime}(1)+O\left(1+\lambda_{n} / \phi_{n}\right)$. By using the same kind of complex integration on the circle $|u-1|=1 / \lambda_{n}$ and the approximation

$$
\begin{aligned}
\exp & \left(\lambda_{n}\left(a(u)-a^{\prime}(1) \log u\right)+\left(b(u)-b^{\prime}(1) \log u\right)\right. \\
& =1+\left(\lambda_{n}\left(a^{\prime \prime}(1)+a^{\prime}(1)\right)+\left(b^{\prime \prime}(1)+b^{\prime}(1)\right)\right) \frac{(u-1)^{2}}{2}+O\left(\lambda_{n}(u-1)^{3}\right)
\end{aligned}
$$

one obtains

$$
\operatorname{Var} X_{n}=\lambda_{n}\left(a^{\prime \prime}(1)+a^{\prime}(1)\right)+O\left(1+\frac{\lambda_{n}}{\phi_{n}}\right) .
$$

If $\sigma^{2}>0$, then $Y_{n} / \sigma$ and $\left(X_{n}-\mathbf{E} X_{n}\right) / \sqrt{\operatorname{Var} X_{n}}$ have the same limiting distribution. Hence, the central limit theorem follows.

In order to obtain tail estimates we proceed as follows. Suppose that $\lambda_{n}=\phi_{n}$. Then we get (similarly as above)

$$
\mathbf{E} e^{t\left(X_{n}-\mathbf{E} X_{n}\right) / \sqrt{\lambda_{n}}}=e^{\frac{\sigma^{2}}{2} t^{2}+O\left(t^{3} / \sqrt{\lambda_{n}}\right)+O\left(t / \sqrt{\lambda_{n}}\right)}\left(1+O\left(\frac{1}{\lambda_{n}}\right)\right) .
$$

Hence, there exist positive constants $c^{\prime}, c^{\prime \prime}, c^{\prime \prime \prime}$ with

$$
\mathbf{E} e^{t\left(X_{n}-\mathbf{E} X_{n}\right) / \sqrt{\lambda_{n}}} \leq c^{\prime} e^{c^{\prime \prime} t^{2}}
$$

for real $t$ with $|t| \leq c^{\prime \prime \prime} \sqrt{\lambda_{n}}$. By a Chernov type argument we get for every $t>0$ the inequality

$$
\operatorname{Pr}\{|Y| \geq \varepsilon\} \leq\left(\mathbf{E} e^{t Y}+\mathbf{E} e^{-t Y}\right) e^{-\varepsilon t} .
$$

We also get (6.16) (for $N=1$ ) by choosing $c_{1}=2 c^{\prime}, t=\varepsilon /\left(2 c^{\prime \prime}\right), c_{2}=1 /\left(4 c^{\prime \prime}\right)$, and $c_{3}=2 c^{\prime \prime} c^{\prime \prime \prime}$.

Now recall that a random vector $\mathbf{Y}$ is normally distributed with zero mean and covariance matrix $\Sigma$ if and only if $\mathbf{a}^{T} \mathbf{Y}=a_{1} Y_{1}+\cdots+a_{N} Y_{N}$ is normally distributed with zero mean and variance $\mathbf{a}^{T} \Sigma \mathbf{a}$. 
Hence, if we assume that a sequence of random vectors $\mathbf{X}_{n}$ satisfies (6.14) then the random variable $X_{n}(\mathbf{a})=\mathbf{a}^{T} \mathbf{X}_{n}$ satisfies $(6.17)$ with $a(u)=A\left(u^{a_{1}}, \ldots, u^{a_{N}}\right)$ and $b(u)=B\left(u^{a_{1}}, \ldots, u^{a_{N}}\right)$. Consequently $X_{n}(\mathbf{a})$ is asymptotically normal with $\mathbf{E} X_{n}(\mathbf{a})=\lambda_{n} \mu+O\left(1+\lambda_{n} / \phi_{n}\right)$ and $\operatorname{Var} X_{n}(\mathbf{a})=\lambda_{n} \sigma^{2}+O\left(1+\lambda_{n} / \phi_{n}\right)$, where

$$
\mu=a^{\prime}(1)=\mathbf{a}^{T} \boldsymbol{\mu} \quad \text { and } \quad \sigma^{2}=a^{\prime}(1)+a^{\prime \prime}(1)=\mathbf{a}^{T} \boldsymbol{\Sigma} \mathbf{a} .
$$

The tail estimate (6.16) can be also derived from the one dimensional case $N=1$. If $\left\|\mathbf{X}_{n}-\mathbf{E} \mathbf{X}_{n}\right\| \geq \varepsilon \sqrt{\lambda_{n}}$ then there exists $j$ with $\left\|X_{n}^{(j)}-\mathbf{E} X_{n}^{(j)}\right\| \geq \varepsilon \sqrt{\lambda_{n}} / \sqrt{N}$. Hence

$$
\operatorname{Pr}\left\{\left\|\mathbf{X}_{n}-\mathbf{E} \mathbf{X}_{n}\right\| \geq \varepsilon \sqrt{\lambda_{n}}\right\} \leq N c_{1} e^{-c_{2} \varepsilon^{2} / N} .
$$

Thus, we also get (6.16) in the multidimensional case. We just have to adjust $c_{1}$ and $c_{2}$.

Putting these preliminaries together we finally get a central limit theorem for random variables that are related to systems of functional equations For convenience we set

$$
\boldsymbol{\mu}=-\frac{x_{0, \mathbf{u}}(\mathbf{1})}{x_{0}(\mathbf{1})}
$$

and define a matrix $\boldsymbol{\Sigma}$ by

$$
\boldsymbol{\Sigma}=-\frac{\mathbf{x}_{\mathbf{0}, \mathbf{u u}}(\mathbf{1})}{\mathbf{x}_{\mathbf{0}}(\mathbf{1})}+\boldsymbol{\mu}^{\mathrm{T}} \boldsymbol{\mu}+\operatorname{diag}(\boldsymbol{\mu}),
$$

where $x=x_{0}(\mathbf{u})$ and $\mathbf{y}=\mathbf{y}_{0}(\mathbf{u})$ are the solutions of the system (6.5) and (6.6).

Theorem 6.6. Suppose that $\mathbf{X}_{n}$ is a sequence of $N$-dimensional random vectors that are defined by (6.12), where $\sum_{n, \mathbf{m}} c_{n, \mathbf{m}} x^{n} \mathbf{u}^{\mathbf{m}}=G(x, \mathbf{y}(x, \mathbf{u}), \mathbf{u})$ and the generating functions $\mathbf{y}(x, \mathbf{u})=\left(y_{j}(x, \mathbf{u})\right)_{1 \leq j \leq N}$ satisfy a system of functional equations of the form (6.7), in which $\mathbf{F}$ satisfies the assumptions of Proposition 6.1.

Then $\mathbf{X}_{n}$ satisfies a central limit theorem of the form

$$
\frac{1}{\sqrt{n}}\left(\mathbf{X}_{n}-\mathbf{E} \mathbf{X}_{n}\right) \rightarrow N(\mathbf{0}, \mathbf{\Sigma})
$$

Furthermore there exist positive constants $c_{1}, c_{2}, c_{3}$ such that

$$
\operatorname{Pr}\left\{\left\|\mathbf{X}_{n}-\mathbf{E} \mathbf{X}_{n}\right\| \geq \varepsilon \sqrt{n}\right\} \leq c_{1} e^{-c_{2} \varepsilon^{2}}
$$

uniformly for $\varepsilon \leq c_{3} \sqrt{n}$.

In what follows we comment on the evaluation of $\boldsymbol{\mu}$ and $\boldsymbol{\Sigma}$. The problem is to extract the derivatives of $x_{0}(\mathbf{u})$. The function $x_{0}(\mathbf{u})$ is the solution of the system (6.5-6.6) and is exactly the location of the singularity of the mapping $x \mapsto \mathbf{y}(x, \mathbf{u})$ when $\mathbf{u}$ is fixed (and close to $\mathbf{1}$ ).

Let $x_{0}(\mathbf{u})$ and $\mathbf{y}_{0}(\mathbf{u})=\mathbf{y}\left(x_{0}(\mathbf{u}), \mathbf{u}\right)$ denote the solutions of $(6.5-6.6)$. Then we have

$$
\mathbf{y}_{0}(\mathbf{u})=\mathbf{F}\left(x_{0}(\mathbf{u}), \mathbf{y}_{0}(\mathbf{u}), \mathbf{u}\right)
$$

Taking derivatives with respect to $\mathbf{u}$ we get

$$
\begin{aligned}
\mathbf{y}_{0, \mathbf{u}}(\mathbf{u}) & =\mathbf{F}_{x}\left(x_{0}(\mathbf{u}), \mathbf{y}_{0}(\mathbf{u}), \mathbf{u}\right) x_{0, \mathbf{u}}(\mathbf{u})+\mathbf{F}_{\mathbf{y}}\left(x_{0}(\mathbf{u}), \mathbf{y}_{0}(\mathbf{u}), \mathbf{u}\right) \mathbf{y}_{0, \mathbf{u}}(\mathbf{u}) \\
& +\mathbf{F}_{\mathbf{u}}\left(x_{0}(\mathbf{u}), \mathbf{y}_{0}(\mathbf{u}), \mathbf{u}\right)
\end{aligned}
$$


where the three terms in $\mathbf{F}$ denote evaluations at $\left(x_{0}(\mathbf{u}), \mathbf{y}_{0}(\mathbf{u}), \mathbf{u}\right)$ of the partial derivatives of $\mathbf{F}$, and not the (total) derivative of the composite function $\mathbf{F}\left(x_{0}(\mathbf{u}), \mathbf{y}_{0}(\mathbf{u}), \mathbf{u}\right)$, and where $x_{\mathbf{u}}$ and $\mathbf{y}_{\mathbf{u}}$ denote the Jacobian of $x$ and $\mathbf{y}$ with respect to $\mathbf{u}$. In particular, for $\mathbf{u}=\mathbf{1}$ we have $x(\mathbf{1})=x_{0}$ and $\mathbf{y}(\mathbf{1})=\mathbf{y}_{0}$ and

$$
\operatorname{det}\left(\mathbf{I}-\mathbf{F}_{\mathbf{y}}\left(x_{0}, \mathbf{y}_{0}, \mathbf{1}\right)\right)=0 .
$$

Since $\mathbf{F}_{\mathbf{y}}$ is a non-negative matrix and the dependency graph is strongly connected there is a unique Perron-Frobenius eigenvalue of multiplicity 1 . Here this eigenvalue equals 1. Thus, $\mathbf{I}-\mathbf{F}_{\mathbf{y}}$ has rank $N-1$ and has (up to scaling) a unique positive left eigenvector $\mathbf{b}^{\mathrm{T}}$ :

$$
\mathbf{b}^{\mathrm{T}}\left(\mathbf{I}-\mathbf{F}_{\mathbf{y}}\left(x_{0}, \mathbf{y}_{0}, \mathbf{1}\right)\right)=\mathbf{0} .
$$

From (6.22) we obtain

$$
\left(\mathbf{I}-\mathbf{F}_{\mathbf{y}}\left(x_{0}, \mathbf{y}_{0}, \mathbf{1}\right)\right) \mathbf{y}_{\mathbf{u}}(\mathbf{1})=\mathbf{F}_{x}\left(x_{0}, \mathbf{y}_{0}, \mathbf{1}\right) x_{\mathbf{u}}(\mathbf{1})+\mathbf{F}_{\mathbf{u}}\left(x_{0}, \mathbf{y}_{0}, \mathbf{1}\right) .
$$

By multiplying $\mathbf{b}^{\mathrm{T}}$ from the left we thus get

$$
\mathbf{b}^{\mathrm{T}} \mathbf{F}_{x}\left(x_{0}, \mathbf{y}_{0}, \mathbf{1}\right) x_{\mathbf{u}}+\mathbf{b}^{\mathrm{T}} \mathbf{F}_{\mathbf{u}}\left(x_{0}, \mathbf{y}_{0}, \mathbf{1}\right)=0
$$

and consequently

$$
\boldsymbol{\mu}=\frac{1}{x_{0}} \frac{\mathbf{b}^{\mathrm{T}} \mathbf{F}_{\mathbf{u}}\left(x_{0}, \mathbf{y}_{0}, \mathbf{1}\right)}{\mathbf{b}^{\mathrm{T}} \mathbf{F}_{x}\left(x_{0}, \mathbf{y}_{0}, \mathbf{1}\right)}
$$

The derivation of $\boldsymbol{\Sigma}$ is more involved. We first define $\mathbf{b}(x, \mathbf{y}, \mathbf{u})$ as the (generalized) vector product ${ }^{7}$ of the $N-1$ last columns of the matrix $\mathbf{I}-\mathbf{F}_{\mathbf{y}}(x, \mathbf{y}, \mathbf{u})$. We define $D(x, \mathbf{y}, \mathbf{u})$ as

$$
D(x, \mathbf{y}, \mathbf{u})=\left(\mathbf{b}^{T}(x, \mathbf{y}, \mathbf{u})\left(\mathbf{I}-\mathbf{F}_{\mathbf{y}}(x, \mathbf{y}, \mathbf{u})\right)\right)_{1}=\operatorname{det}\left(\mathbf{I}-\mathbf{F}_{\mathbf{y}}(x, \mathbf{y}, \mathbf{u})\right),
$$

where the subindex denotes the first coordinate. In particular we have

$$
D(x(\mathbf{u}), \mathbf{y}(\mathbf{u}), \mathbf{u})=0 .
$$

Then from

$$
\begin{aligned}
\left(\mathbf{I}-\mathbf{F}_{\mathbf{y}}\right) \mathbf{y}_{\mathbf{u}} & =\mathbf{F}_{x} x_{\mathbf{u}}+\mathbf{F}_{\mathbf{u}}, \\
-D_{\mathbf{y}} \mathbf{y}_{\mathbf{u}} & =D_{x} x_{\mathbf{u}}+D_{\mathbf{u}}
\end{aligned}
$$

we can calculate $\mathbf{y}_{\mathbf{u}}$. (The first system has rank $N-1$, this means that we can skip the first equation. This reduced system is then completed to a regular system by appending the second equation (6.24).)

We now set

$$
\begin{aligned}
d_{1}(\mathbf{u}) & =d_{1}(x(\mathbf{u}), \mathbf{y}(\mathbf{u}), \mathbf{u})=\mathbf{b}(x(\mathbf{u}), \mathbf{y}(\mathbf{u}), \mathbf{u})^{\mathrm{T}} \mathbf{F}_{x}(x(\mathbf{u}), \mathbf{y}(\mathbf{u}), \mathbf{u}) \\
\mathbf{d}_{2}(\mathbf{u}) & =\mathbf{d}_{2}(x(\mathbf{u}), \mathbf{y}(\mathbf{u}), \mathbf{u})=\mathbf{b}(x(\mathbf{u}), \mathbf{y}(\mathbf{u}), \mathbf{u})^{\mathrm{T}} \mathbf{F}_{\mathbf{u}}(x(\mathbf{u}), \mathbf{y}(\mathbf{u}), \mathbf{u}) .
\end{aligned}
$$

By differentiating equation (6.23) we get

$$
x_{\mathbf{u u}}(\mathbf{u})=-\frac{\left(d_{1 x} x_{\mathbf{u}}+d_{1 \mathbf{y}} \mathbf{y}_{\mathbf{u}}+d_{1 \mathbf{u}}\right) x_{\mathbf{u}}+\left(\mathbf{d}_{2 x} x_{\mathbf{u}}+\mathbf{d}_{2 \mathbf{y}} \mathbf{y}_{\mathbf{u}}+\mathbf{d}_{2 \mathbf{u}}\right)}{d_{1}},
$$

where $d_{1 x}, d_{1 \mathbf{y}}, d_{1 \mathbf{u}}, \mathbf{d}_{2 x}, \mathbf{d}_{2 \mathbf{y}}, \mathbf{d}_{2 \mathbf{u}}$ denote the respective partial derivatives, and where we have omitted the dependence on $\mathbf{u}$. With the knowledge of $x_{0}, \mathbf{y}_{0}$ and $\mathbf{y}_{\mathbf{u}}(\mathbf{1})$ we can now evaluate $x_{\mathbf{u u}}$ at $\mathbf{u}=\mathbf{1}$ and we compute $\boldsymbol{\Sigma}$ from (6.18).

\footnotetext{
${ }^{7}$ More precisely, this is the wedge product combined with the Hodge duality.
} 
We finally state a useful variant of a central limit theorem for random variables that are defined with the help of generating functions.

Theorem 6.7. Suppose that a sequence of $N$-dimensional random vectors $\mathbf{X}_{n}$ satisfies

$$
\mathbf{E} \mathbf{u}^{\mathbf{X}_{n}}=\frac{c_{n}(\mathbf{u})}{c_{n}(\mathbf{1})}
$$

where $c_{n}(\mathbf{u})$ is the coefficient of $x^{n}$ of an analytic function

$$
f(x, \mathbf{u})=\sum_{n \geq 0} c_{n}(\mathbf{u}) x^{n},
$$

that has a local singular representation of the form

$$
f(x, \mathbf{u})=g(x, \mathbf{u})+h(x, \mathbf{u})\left(1-\frac{x}{\rho(\mathbf{u})}\right)^{\alpha}
$$

for some real $\alpha \in \mathbb{R} \backslash \mathbb{N}$ and functions $g(x, \mathbf{u}), h(x, \mathbf{u}) \neq 0$, and $\rho(\mathbf{u}) \neq 0$ that are analytic around $x=x_{0}>0$ and $\mathbf{u}=\mathbf{1}$. Further we assume that $x=\rho(\mathbf{u})$ is the only singularity of $f(x, u)$ on the disc $|x| \leq|\rho(\mathbf{u})|$ if $\mathbf{u}$ is sufficiently close to $\mathbf{1}$ and that there exists an analytic continuation of $f(x, u)$ to the region $|x|<|\rho(u)|+\delta$, $|x-\rho(\mathbf{u})|>\varepsilon$ for some $\delta>0$ and $\varepsilon>0$.

Then $\mathbf{X}_{n}$ satisfies a central limit theorem of the form (6.19) and tail estimates of the form (6.20) with $\mathbf{E} \mathbf{X}_{n}=\boldsymbol{\mu} n+O(1)$ and $\mathbf{C o v} \mathbf{X}_{n}=\boldsymbol{\Sigma} n+O(1)$, where

$$
\boldsymbol{\mu}=-\frac{\rho_{\mathbf{u}}(\mathbf{1})}{\rho(\mathbf{1})}
$$

and

$$
\boldsymbol{\Sigma}=-\frac{\rho_{\mathbf{u u}}(\mathbf{1})}{\rho(\mathbf{1})}+\boldsymbol{\mu}^{\mathrm{T}} \boldsymbol{\mu}+\operatorname{diag}(\boldsymbol{\mu})
$$

Proof. By the transfer lemma in [8] we get the asymptotic expansion

$$
c_{n}(\mathbf{u})=\frac{h(\rho(\mathbf{u}), \mathbf{u})}{\Gamma(-\alpha)} n^{-\alpha-1} \rho(\mathbf{u})^{-n}\left(1+O\left(\frac{1}{n}\right)\right)
$$

that is uniform for $\mathbf{u}$ in a complex neighbourhood of $\mathbf{u}=\mathbf{1}$. Hence,

$$
\begin{aligned}
\mathbf{E} \mathbf{u}^{\mathbf{X}_{n}} & =\frac{c_{n}(\mathbf{u})}{c_{n}(\mathbf{1})} \\
& =\frac{h(\rho(\mathbf{u}), \mathbf{u})}{h(\rho(\mathbf{1}), \mathbf{1})}\left(\frac{\rho(\mathbf{1})}{\rho(\mathbf{u})}\right)^{n}\left(1+O\left(\frac{1}{n}\right)\right)
\end{aligned}
$$

and consequently the result follows from Proposition 6.4.

\section{Proof of Theorem 1.1}

7.1. 2-Connected Outerplanar Graphs. We fix some $k \geq 1$ and let $X_{n}^{(k)}$ denote the random variable that counts the number of vertices of degree $k$ in a random 2-connected outerplanar vertex labelled graph of size $n$. In particular, if $b_{n, \nu}$ is the number of 2-connected outerplanar vertex labelled graph of size $n$ with exactly $\nu$ vertices of degree $k$ and

$$
B_{d=k}(x, u)=\sum_{n \geq 0} \frac{b_{n}(u)}{n !} x^{n}
$$


is the corresponding generating function then

$$
\mathbf{E} u^{X_{n}^{(k)}}=\frac{b_{n}(u)}{b_{n}(1)}
$$

where

$$
b_{n}(u)=\left[x^{n}\right] B_{d=k}(x, u)=\sum_{\nu \geq 0} b_{n, \nu} u^{\nu} .
$$

In Section 2 we have shown that the derivative

$$
\frac{\partial B_{d=k}(x, u)}{\partial x}=\sum_{n \geq 0}(n+1) b_{n+1}(u) x^{n}
$$

can be represented in terms of the functions $B_{j}^{\bullet}\left(x, z_{1}, \ldots, z_{k}, z_{\infty}\right)$; see (2.3). Furthermore, these functions are linear combinations of the functions $A_{i j}\left(x, z_{1}, \ldots, z_{k}, z_{\infty}\right)$; see Lemma 2.4. Finally, the functions $A_{i j}$ satisfy a positive and strongly connected system of equations (see Lemma 2.2).

Since the coefficient of $x^{n}$ of $\frac{\partial B_{d=k}(x, u)}{\partial x}$ equals $(n+1) b_{n+1}(u)$ we have

$$
\frac{(n+1) b_{n+1}(u)}{(n+1) b_{n+1}(1)}=\frac{b_{n+1}(u)}{b_{n+1}(1)}=\mathbf{E} u^{X_{n+1}^{(k)}}
$$

Hence, by applying Theorem 6.6 it follows directly that $X_{n+1}^{(k)}$ satisfies a central limit theorem with linear expected value and variance and quadratic exponential tail estimates. Of course, this implies the same properties for $X_{n}^{(k)}$ and proves Theorem 1.1 for 2-connected outerplanar graphs.

Alternatively we can use Proposition 6.1 and its corollary to derive that $\frac{\partial B_{d=k}(x, u)}{\partial x}$ has a local representation of the form

$$
\frac{\partial B_{d=k}(x, u)}{\partial x}=g(x, u)-h(x, u) \sqrt{1-\frac{x}{\rho(u)}} .
$$

Hence, by Lemma 5.2 , we get a local representation for $B_{d=k}(x, u)$ of the form

$$
B_{d=k}(x, u)=\bar{g}(x, u)+\bar{h}(x, u)\left(1-\frac{x}{\rho(u)}\right)^{3 / 2} .
$$

Thus, the central limit theorem follows from Theorem 6.7, too.

Note that Theorems 6.6 and 6.7 also apply for random vectors. Thus, if we set $z_{j}=u_{j}$ for $1 \leq j \leq k$ and $z_{\infty}=1$ in the generating function $B\left(x, z_{1}, \ldots, z_{k}, z_{\infty}\right)$, we also get a multivariate central limit theorem for then random vector $\left(X_{n}^{(1)}, \ldots, X_{n}^{(k)}\right)$.

Finally, we can also use Theorem 6.6 to prove that the number of vertices of given degree in random dissections of size $n$ satisfies a central limit theorem.

7.2. Connected Outerplanar Graphs. The proof of Theorem 1.1 for connected outerplanar graphs is very similar to the case of 2-connected outerplanar graphs.

We recall that the derivative

$$
\frac{\partial C_{d=k}(x, u)}{\partial x}=\sum_{n \geq 0}(n+1) c_{n+1}(u) x^{n}
$$

can be represented as a linear combination of the functions $C_{j}^{\bullet}\left(x, z_{1}, \ldots, z_{k}, z_{\infty}\right)$ (see $(2.5)$ ) that satisfy a positive and strongly connected system of equations, see Lemma 2.6. Note that the system of equations given in Lemma 2.6 uses the functions 
$B_{j}^{\bullet}\left(x, z_{1}, \ldots, z_{k}, z_{\infty}\right)$, that have a square-root singularity if the $z_{j}$ are sufficiently close to 1 ; recall that $B^{\prime}(x)$ and $B_{j}^{\bullet}(x, 1, \ldots, 1)$ have a square-root singularity at $\rho_{1}=3-2 \sqrt{2}$. In order to apply Theorem 6.6 we have to check that the critical point of the system (2.5) does not interfere with the square-root singularity of $B_{j}^{\bullet}$.

Since $f(x)=x C^{\prime}(x)$ satisfies the equation $f(x)=e^{B^{\prime}(f(x))}$, the function $\psi(u)=u e^{-B^{\prime}(u)}$ is the functional inverse function of $f(x)$. We know from [3] that there exists $\rho_{2}=0.1688 \cdots<\rho_{1}$ with $\psi^{\prime}(u)=0$. This implies that the radius of convergence of $f(x)$ and consequently that of $C^{\prime}(x)$ is given by $\rho=0.1366 \cdots$. It satisfies $\rho C^{\prime}(\rho)=\rho_{2}$, and $C^{\prime}(x)$ has a square root singularity with a local representation of the form

$$
C^{\prime}(x)=g_{2}(x)-h_{2}(x) \sqrt{1-\frac{x}{\rho}}
$$

where $g(x)$ and $h(x)$ are analytic around $x=\rho_{2}$, see also the remark following Lemma 2.5.

All functions $C_{j}^{\bullet}(x, 1, \ldots, 1)$ have the same singularity $\rho$. Thus, the corresponding system of equation (6.5)-(6.6) is satisfied for $x=\rho$ and $\mathbf{y}=$ $\left(C_{j}^{\bullet}(\rho, 1, \ldots, 1)\right)_{j \in\{1, \ldots, k, \infty\}}$ and $\mathbf{u}=\left(z_{1}, \ldots, z_{k}, z_{\infty}\right)=\mathbf{1}$. By the implicit function theorem there is also a solution if $z_{1}, \ldots, z_{k}$ vary locally around 1 ; we fix $z_{\infty}=1$ so that $x$ and $z_{1}, \ldots, z_{k}$ can vary independently. Thus, if $z_{1}, \ldots, z_{k}$ are sufficiently close to 1 , then all functions $C_{j}^{\bullet}\left(x, z_{1}, \ldots, z_{k}, 1\right)$ have again a square-root singularity.

Summing up, we can again apply Theorem 6.6 and obtain Theorem 1.1 for connected outerplanar graphs, too. However, the degree distribution is different from that of 2-connected outerplanar graphs.

Further, we also get a multivariate central limit theorem for the random vectors $\left(X_{n}^{(1)}, \ldots, X_{n}^{(k)}\right)$.

7.3. All Outerplanar Graphs. From the discussion in the preceding section it follows that the partial derivative $\frac{\partial C_{d=k}(x, u)}{\partial x}$ has a local representation of the form

$$
\frac{\partial C_{d=k}(x, u)}{\partial x}=g_{2}(x, u)-h_{2}(x, u) \sqrt{1-\frac{x}{\rho(u)}} .
$$

Hence, by Lemma 5.2 we get the representation

$$
C_{d=k}(x, u)=g_{3}(x, u)+h_{3}(x, u)\left(1-\frac{x}{\rho(u)}\right)^{3 / 2} .
$$

Consequently we obtain a representation for $G_{d=k}(x, u)$ of the form

$$
G_{d=k}(x, u)=e^{C_{d=k}(x, u)}=g_{4}(x, u)+h_{4}(x, u)\left(1-\frac{x}{\rho(u)}\right)^{3 / 2} .
$$

Thus, the central limit theorem follows from Theorem 6.7.

\section{Proof of Theorem 1.2}

The proof is more involved than that of Theorem 1.1. Due to the relation between series-parallel networks and 2-connected series-parallel graphs, we have to take into account the number of edges. 
8.1. 2-Connected Series-Parallel Graphs. We first observe that the function $D_{i j}$ and $S_{i j}$ satisfy a positive and stongly connected system of equations (Lemma 3.2), so that by Proposition 6.1 all these functions have a common squareroot singularity of the kind

$$
g\left(x, y, z_{1}, \ldots, z_{k}, z_{\infty}\right)-h\left(x, y, z_{1}, \ldots, z_{k}, z_{\infty}\right) \sqrt{1-\frac{x}{\rho\left(y, z_{1}, \ldots, z_{k}, z_{\infty}\right)}} .
$$

Hence, by Lemma 5.1 the same is true for all function $B_{i j}$ and consequently for

$$
2 y \frac{\partial B}{\partial y}=\sum_{i, j \in\{1, \ldots, k, \infty\}} B_{i j} .
$$

From Proposition 5.4 it follows that $B$ has a singularity of the kind

$$
\bar{g}\left(x, y, z_{1}, \ldots, z_{k}, z_{\infty}\right)+\bar{h}\left(x, y, z_{1}, \ldots, z_{k}, z_{\infty}\right)\left(1-\frac{x}{\rho\left(z_{1}, \ldots, z_{k}, z_{\infty}\right)}\right)^{3 / 2} .
$$

As consequence, by applying Theorem 6.7 we derive Theorem 1.2 for the 2connected case. Notice that Theorem 6.7 is essential in this case; in fact, it was derived precisely for the needs of this application.

Note that $B_{1}, \ldots B_{k}$ have also a square-root singularity of the kind (8.1), since they can be expressed with the help of the function $B_{i j}$. Interestingly, $B_{\infty}$ has the same kind of singularity. This follows from formula

$$
B_{\infty}=x \frac{\partial B}{\partial x}-\sum_{i=1}^{k} B_{i}
$$

since $\frac{\partial B}{\partial x}$ has also a square-root singularity of the kind (8.1).

8.2. Connected Series-Parallel Graphs. Here we can proceed exactly as in the outerplanar case. The essential point is to observe that the singularity of $B_{i}^{\bullet}$ does not interfere with the singularity of $C_{j}^{\bullet}$; this is proved also in [3]. As mentioned in the introduction, the equations from Lemma 2.6 linking the $B_{j}^{\bullet}$ and the $C_{j}^{\bullet}$ apply here as well.

8.3. All Series-Parallel Graphs. As in the outerplanar case it follows that $C_{d=k}(x, u)$ has a singularity of the kind (7.1). Hence, the same follows for $G_{d=k}(x, u)$ and, thus, we can apply Theorem 6.7 .

\section{Proof of Theorem 1.3}

The limit distribution for the number of vertices of degree 1 in planar graphs was determined in [10], where it is shown that a central limit theorem with linear mean and variance holds. This follows from Theorem 4 in [10], applied to the case where $H$ is a single edge. It is easy to extend this result to get corresponding tail estimates as proposed. Thus, we concentrate on the case $k=2$.

9.1. 2-Connected Planar Graphs. We recall that

$$
\begin{aligned}
B_{p}\left(x, y, z_{1}, z_{2}, z_{\infty}\right) & =B\left(x, y, z_{1}, z_{2}, z_{\infty}\right)+H_{p}\left(x, D\left(x, y, z_{1}, z_{2}, z_{\infty}\right)\right) \\
& =B_{p}\left(x, \phi\left(x, D\left(x, y, z_{1}, z_{2}, z_{\infty}\right)\right)\right) \\
& +B\left(x, y, z_{1}, z_{2}, z_{\infty}\right)-B\left(x, \phi\left(x, D\left(x, y, z_{1}, z_{2}, z_{\infty}\right)\right)\right) .
\end{aligned}
$$


In [10] it was shown that $B_{p}(x, y)$ has a singular expansion of the form

$$
B_{p}(x, y)=B_{0}(y)+B_{2}(y) X^{2}+B_{4}(y) X^{4}+B_{5}(y) X^{5}+B_{6}(y) X^{6}+\cdots,
$$

where $X=\sqrt{1-x / R(y)}$, where $R(1)=0.03819 \cdots$. Alternatively this can be rewritten as

$$
B_{p}(x, y)=g(x, y)+h(x, y)\left(1-\frac{x}{R(y)}\right)^{\frac{5}{2}} .
$$

Further note that $B\left(x, y, z_{1}, z_{2}, z_{\infty}\right), B(x, y)=B(x, y, 1, \ldots, 1)$, and $\phi\left(x, D\left(x, y, z_{1}, z_{2}, z_{\infty}\right)\right)$ are analytic around $x=R(1), y=1$ and $z_{j}=1$, $j \in\{1, \ldots, k, \infty\}$. Hence, $B_{p}\left(x, y, z_{1}, z_{2}, z_{\infty}\right)$ can be represented as

$$
\begin{aligned}
B_{p}\left(x, y, z_{1}, z_{2}, z_{\infty}\right)= & g_{2}\left(x, y, z_{1}, z_{2}, z_{\infty}\right)+ \\
& h_{2}\left(x, y, z_{1}, z_{2}, z_{\infty}\right)\left(1-\frac{x}{\bar{R}\left(y, z_{1}, z_{2}, z_{\infty}\right)}\right)^{5 / 2},
\end{aligned}
$$

where $g_{2}, h_{2}$, and $\bar{R}$ are analytic functions with

$$
\begin{aligned}
g_{2}(x, y, 1,1,1) & =g(x, y), \\
h_{2}(x, y, 1,1,1) & =h(x, y), \\
\bar{R}(y, 1,1,1) & =R(y) .
\end{aligned}
$$

We can apply Theorem 6.7 with $\alpha=\frac{5}{2}$ and obtain a proof of Theorem 1.3 in the 2-connected case.

9.2. Connected Planar Graphs. From (9.1) it follows that $B_{j}^{\bullet}\left(x, y, z_{1}, z_{2}, z_{\infty}\right)$ have a singularity of the kind (8.2). Hence, the function $F(x, y, u)$ from Lemma 4.4 has also a singularity of that kind. Note that $F(x, y, 1)=e^{B^{\bullet}(x, x y)}$. Furthermore, for $u=1$ it is known that the the singularity of $C^{\bullet}$ interfers with the singulartiy of $B^{\bullet}$, compare with [10]. Hence all assumptions of Proposition 6.3 are satisfied and it follows that $C^{\bullet}(x, 1,1, u, 1)$ has a singular expansion of the form (8.2). Of course, with help of Theorem 6.7 we get Theorem 1.3 in the connected case, too.

9.3. All Planar Graphs. The only difference between the case of planar graphs and the two previous cases (outerplanar and series-parallel graphs) is that the singular behaviour of $C_{d=2}(x, u)$ resp. $G_{d=2}(x, u)$ is of the form

$$
g_{5}(x, u)+h_{5}(x, u)\left(1-\frac{x}{\rho(u)}\right)^{5 / 2} .
$$

\section{REFERENCES}

[1] E. A. Bender, Central and Local Limit Theorems Applied to Asymptotic Enumeration, J. Combinat. Theory Ser. A 15 (1973), 91-111.

[2] E. A. Bender and L. B. Richmond, Central and local limit theorems applied to asymptotic enumeration II: multivariate generating functions, J. Combin. Theory Ser. A 34 (1983), 255265.

[3] M. Bodirsky, O. Giménez, M. Kang, and M. Noy, Enumeration and limit laws for seriesparallel graphs, Europ. J. Combinatorics 28 (2007), 2091-2105.

[4] F. Chyzak, M. Drmota, T. Klausner, G. Kok, The Distribution of Patterns in Random Trees, Combinatorics Probability and Computing 17 (2008), 21-59.

[5] M. Drmota, Systems of functional equations, Random Struct. Algorithms 10 (1997), 103-124.

[6] M. Drmota, O. Giménez and M. Noy, Degree distribution in random planar graphs (in preparation). 
[7] P. Flajolet, M. Noy, Analytic Combinatorics of Non-crossing Configurations, Discrete Math. (1999).

[8] P. Flajolet and A. M. Odlyzko, Singularity analysis of generating functions, SIAM J. Discrete Math. 3 (1990), 216-240.

[9] P. Flajolet and R. Sedgewick, Analytic Combinatorics, web-edition, 809+xii pages, (available from the authors' web sites). To be published in 2008 by Cambridge University Press.

[10] O. Giménez, M. Noy, The number of planar graphs and properties of random planar graphs, 2005 International Conference on Analysis of Algorithms, DMTCS Proceedings, vol. AD, 2005, pp. 147-156. Full version available at http://arxiv.org/abs/math/0501269

[11] H.-K. Hwang, Théoremes limites pour les structures combinatoires et les fonctions arithmetiques, PhD thesis, École Polytechnique, Dec. 1994.

[12] H.-K. Hwang, Large deviations for combinatorial distributions. I. Central limit theorems, Ann. Appl. Prob. 6 (1996), 297-319.

[13] L. Kaup and B. Kaup, Holomorphic Functions of Several Variables, Studies in Mathematic, de Gruyter, Berlin, 1983.

[14] C. McDiarmid, Random graphs on surfaces, CRM preprint 2006, available at http://www.crm.cat/Publications/06/Pr722.pdf

[15] T. R. S. Walsh, Counting labelled three-connected and homeomorphically irreducible twoconnected graphs, J. Combin. Theory Ser. B 32 (1982), 1-11. 